\title{
Comparative functional genomic analysis of Alzheimer's affected and naturally aging brains
}

\author{
Yi-Shian Peng ${ }^{1}$, Chia-Wei Tang ${ }^{1}$, Yi-Yun Peng ${ }^{1}$, Hung Chang ${ }^{1}$, Chien-Lung Chen ${ }^{2}$, Shu-Lin Guo ${ }^{3,4}{ }^{4}$ Li-Ching Wu ${ }^{1}$, \\ Min-Chang Huang ${ }^{5}$, Hoong-Chien Lee ${ }^{\text {Corresp. } 1,5}$ \\ 1 Department of Biomedical Sciences and Engineering, National Central University, Taoyuan, Taiwan \\ 2 Department of Nephrology, Landseed Hospital, Taoyuan, Taiwan \\ 3 Department of Anesthesiology, Cathay General Hospital, Taipei, Taiwan \\ 4 Department of Anesthesiology, Tri-Service General Hospital and National Defense Medical Center, Taipei, Taiwan \\ 5 Department of Physics, Chung Yuan Christian University, Taoyuan, Taiwan \\ Corresponding Author: Hoong-Chien Lee \\ Email address: hclee12345@gmail.com
}

Background. Alzheimer's disease (AD) is a prevalent progressive neurodegenerative human disease whose cause remains unclear. Numerous initially highly hopeful anti-AD drugs based on the amyloid- $\beta(A \beta)$ hypothesis of $A D$ have failed recent late-phase tests. Natural aging $(A G)$ is a high-risk factor for AD. Here, we aim to gain insights in AD that may lead to its novel therapeutic treatment through conducting meta-analyses of gene expression microarray data from AG and AD-affected brain. Methods. Five sets of gene expression microarray data from different regions of AD-affected brain, and one set from $A G$, were meta-analyzed by means of the application of the methods of differentially expressed genes and differentially co-expressed gene pairs for the identification of putatively disrupted biological pathways and associated abnormal molecular contents. Results. Brain-region specificity among ALZ cases and AG-ALZ differences in gene expression and in KEGG pathway disruption were identified. Strong heterogeneity in AD signatures among the five brain regions was observed: HC/PC/SFG showed clear and pronounced AD signatures, MTG moderately so, and EC showed essentially none. There were stark differences between ALZ and AG. OXPHOS and Proteasome were the most disrupted pathways in HC/PC/SFG, while AG showed no OXPHOS disruption and relatively weak Proteasome disruption in AG. Metabolic related pathways including TCA cycle and Pyruvate metabolism were disrupted in ALZ but not in AG. Three pathogenic infection related pathways were disrupted in ALZ. Many cancer and signaling related pathways were shown to be disrupted AG but far less so in ALZ, and not at all in HC. We identified 54 "ALZ-only" differentially expressed genes, all down-regulated and which, when used to augment the gene list of the KEGG AD pathway, made it significantly more AD-specific. 
1 Comparative functional genomic analysis of Alzheimer's 2 affected and naturally aging brains

3

4 Yi-Shian Peng*1, Chia-Wei Tang ${ }^{1}$, Yi-Yun Peng ${ }^{1}$, Hung Chang ${ }^{1}$, Chien-Lung Chen ${ }^{2}$, Shu-Lin

$5 \mathrm{Guo}^{3,4}$, Li-Ching $\mathrm{Wu}^{1}$, Min-Chang Huang ${ }^{5}$, Hoong-Chien Lee*1,5

6

$7{ }^{1}$ Department of Biomedical Sciences and Engineering, National Central University, Taoyuan,

8 Taiwan 32001

9 2Department of Nephrology, Landseed Hospital, Taoyuan, Taiwan 324

$10{ }^{3}$ Department of Anesthesiology, Cathay General Hospital, Taipei, Taiwan 106

$11{ }^{4}$ Department of Anesthesiology, Tri-Service General Hospital and National Defense Medical

12 Center, Taipei, Taiwan 114

13 5 Department of Physics, Chung Yuan Christian University, Taoyuan, Taiwan 32023

\section{*Corresponding authors}

16 Hoong-Chien Lee ${ }^{1,5}$

17300 Zhongda Road, Zhongli District Taoyuan City, 32001, Taiwan

18 E-mail: hclee12345@gmail.com

19

20

Yi-Shian Peng ${ }^{1}$

21

300 Zhongda Road, Zhongli District Taoyuan City, 32001, Taiwan

E-mail: bim962511@gmail.com

23

24

25

26 
27 Abstract

28 Background. Alzheimer's disease (AD) is a prevalent progressive neurodegenerative human

29 disease whose cause remains unclear. Numerous initially highly hopeful anti-AD drugs based on 30 the amyloid- $\beta(\mathrm{A} \beta)$ hypothesis of $\mathrm{AD}$ have failed recent late-phase tests. Natural aging (AG) is a

31 high-risk factor for AD. Here, we aim to gain insights in AD as well as AG that may lead to

32 methods for the early detection of $\mathrm{AD}$ and possibly its novel therapeutic treatment through 33 conducting detailed analyses of gene expression microarray data from AG and AD-affected 34 brain.

35 Methods. Five sets of gene expression microarray (ALZ) data from different regions of AD36 affected brain, and one set from AG, were analyzed by means of the application of the methods 37 of differentially expressed genes and differentially co-expressed gene pairs for the identification 38 of putatively disrupted KEGG pathways and associated abnormal molecular contents.

39 Results. Brain-region specificity among ALZ cases and AG-ALZ differences in gene expression 40 and in KEGG pathway disruption were identified. Strong heterogeneity in AD signatures among 41 the five brain regions was observed: $\mathrm{HC} / \mathrm{PC} / \mathrm{SFG}$ showed clear and pronounced $\mathrm{AD}$ signatures, 42 MTG moderately so, and EC showed essentially none. There were stark differences between 43 ALZ and AG. OXPHOS and Proteasome were the most disrupted pathways in $\mathrm{HC} / \mathrm{PC} / \mathrm{SFG}$, 44 while AG showed no OXPHOS disruption and relatively weak Proteasome disruption in AG. 45 Metabolic related pathways including TCA cycle and Pyruvate metabolism were disrupted in 46 47 ALZ but not in AG. Three pathogenic infection related pathways were disrupted in ALZ. Many cancer and signaling related pathways were shown to be disrupted AG but far less so in ALZ, and not at all in HC. We identified 54 "ALZ-only" differentially expressed genes, all downregulated and which, when used to augment the gene list of the KEGG AD pathway, made it significantly more AD-specific.

51

Keywords: Alzheimer's disease, aging, novel AD target genes, KEGG pathways, OXPHOS, proteasome, pyruvate metabolism, TCA cycle, pathogen infection, tau and A $\beta$ pathologies, antimicrobial protection hypothesis

\section{Introduction}


57 Aging (AG), or senescence, is a natural process that leads to deterioration in biological, 58 physical, mental, and neurodegenerative disorders including dementia [1]. Alzheimer's disease 59 (AD), a neurodegenerative disorder with millions of individuals affected worldwide, is the most 60 common type of dementia [2].

61 The etiology of AD is not well understood, but about $70 \%$ of the risk for AD is thought to 62 be involving many genes. In the last 25 years, the amyloid cascade, or amyloid- $\beta$ (A $\beta)$, 63 hypothesis $[3,4]$, which holds that $\mathrm{A} \beta$ aggregation in the brain is a main causative factor of $\mathrm{AD}$ 64 and mutations in presenilin 1 (PSEN1) [5], presenilin 2 (PSEN2) [6], amyloid precursor protein 65 66 $(A P P)$ [7], and apolipoprotein $\mathrm{E}(A P O E)$ [8] are responsible for $\mathrm{A} \beta$ production, has been widely accepted, and depletion of $A \beta$ supply or obstruction of $A \beta$ production through targeting the four genes has been a mainstay of anti-AD drug design strategy. The four genes, together with tau protein $(M A P T)$ [9] (thought to be responsible for neurofibrillary tangles, a second main hypothesis on AD pathology [10]) have been termed the five AD "culprit" genes. PENSI and PENS2 are parts of the $\gamma$-secretase complex, the enzyme (together with $\beta$-secretase 1 , or BACE1) that cleaves $A P P$ to produce $\mathrm{A} \beta$, and $A P O E$ enhances proteolytic breakdown of $\mathrm{A} \beta$. The last few years have seen late-phase failures of the trials of many of these drugs: Semagacestat [11] is an inhibitor targeting $\gamma$-secretase to obstruct $A \beta$ production, Atabecestat [12] and Verubecestat [13] inhibit BACE1 ( $\beta$-secretase), and Aducanumab [14], Bapineuzumab [15], Solanezumab [15] and Crenezumab [16] are humanized monoclonal antibodies designed to target A $\beta$. Reasons for the test failures [17] are not known and the underlying pathophysiology of AD remains unclear.

Numerous studies have reported genetic links between $\mathrm{AD}$ and $\mathrm{AG}$, including that $\mathrm{AD}$ and AG share a common set of declining synaptic genes [18], and that genes related to mitochondrial metabolism and energy production [19], and genes involved in neuronal calcium dependent signaling [20], are significantly downregulated in both AD and AG. The identification of differentially expressed genes (DEGs) [21] has been widely used in the study of complex disorders, including AD [22]. Such studies have implicated as possible causes for AD mitochondrial and DNA damage [23], inflammatory response [24], ubiquitin-proteasome dysfunction [25], and others. Recently the method of differential co-expression (DCE) analysis has been proposed as suitable for understanding biological signatures in complex diseases [26].

Aging has long been recognized as a major rick for neurological disorders, including AD. 
88

89

90

91

92

93

94

95

96

97

98

99

100

101

102

103

104

105

106

107

108

109

110

111

112

113

\section{1}

114 Materials and Methods

115 Gene expression microarray data source

116 Six sets of gene expression microarray data (the six cases) were selected from Gene 117 Expression Omnibus (GEO) [27] (http://www.ncbi.nlm.nih.gov/geo/). (a) Five sets of ALZ data 118 (GEO accession no. GSE5281) taken from five regions of the brain selected for being known to

differentiating between normal AG and the early onset of AD is not. The ability to detect $\mathrm{AD}$ at its early stages offers the best possibility of treatment, either slowing or arresting its progress and, hopefully it the future, reversing it. In this study, our goal is to identify dysfunctional signatures of AG and AD separately, to examine how they differ, and to gain insights into recognizing signatures of early onset of $\mathrm{AD}$. The materials used for this study were six sets of whole-genome gene expression microarray data, one set for AG (brain tissues from 70 years and older versus 40 years old and younger) and five sets for ALZ (tissues from five brain regions entorhinal cortex (EC), hippocampus (HC), medial temporal gyrus (MTG), posterior cingulate (PC), superior frontal gyrus (SFG) - of 65 years and older AD patients versus age-matched healthy controls). For clarity, we use ALZ instead of AD when specifically referring to the AD datasets. From each of the six datasets (or cases) we curated sets of DEG and interacting differentially co-expressed (IDCE) genes pairs. The method of gene set enrichment and KEGG pathways were employed on these curated gene sets to identify putatively disrupted biological pathways (or functions). The enriched contents of pathways were analyzed in detail comparatively case wise. Our analysis revealed strong heterogeneity in AD signatures among the five brain regions, with $\mathrm{HC}, \mathrm{PC}$, and SFG showing clear and pronounced AD signature, MTG moderately so, and EC showing almost none. There was stark difference between ALZ and AG, the most notable being the very strong OXPHOS and Proteasome disruptions in $\mathrm{HC} / \mathrm{PC} / \mathrm{SFG}$, but no OXPHOS disruption and only weak Proteasome disruption in AG. Our result is consistent with the Antimicrobial Protection Hypothesis of AD. We identified 54 "ALZ-only" differentially expressed genes, all down regulated and which, when used to augment the gene list of the KEGG AD pathway, made it significantly more AD-specific. 
119 be differentially vulnerable to the histopathological and metabolic features of $\operatorname{AD}[28,29]$ :

120 entorhinal cortex (EC, from Brodmann's areas 28 and 34), hippocampus (HC), medial temporal 121 gyrus (MTG, BA 21 and 37 and proximate BA 22), posterior cingulate (PC, BA 23 and 31), and

122 superior frontal gyrus (SFG, mostly BA 8). Data from a sixth region, primary visual cortex

123 (VCX; BA 17) were not included in this analysis. (b) One set of AG data (GEO project accession 124 no. GSE53890) [30] taken from prefrontal cortex (BA 9, 10, 11, 12, 46, and 47) of normal

125 healthy cohort 26 to 106 years of age. From the AG data two groups were selected, the young or 126 control group, age $<40$, and the old or test group, age $>70$. All microarray data were on the 127 platform Affymetrix U133 plus 2.0 (Supplementary Table 1).

128

129

130

131

132

133

134

135

136

137

138

139

140

141

142

143

144

145

146

147

\section{Database on protein-protein interaction}

Protein-protein interaction (PPI) information on 12,231 human protein entries and 74,236 interactions (non-redundant) were downloaded from Human Protein Reference Database (HPRD) [31] (http://www.hprd.org/) and Uniprot [32] (http://www.uniprot.org/) and used in the construction of IDCE pairs.

\section{KEGG database on biological functions and pathways}

The Kyoto Encyclopedia of Genes and Genomes (KEGG), a database for biological categories, including biological pathways, was used for querying the functional enrichment of gene sets [33].

\section{Two setss of known AD target genes from AlzGene and AlzBase}

Two types of known AD genes were used in this study, AlzGene [34] (Alzforum; https://www.alzforum.org/) and AlzBase [35] (http://alz.big.ac.cn/alzBase/home). AlzGene is a collection of published Alzheimer's disease genetic association studies aimed to include GWAS meta-analysis results. It contains information on 8,246 GWAS entries and 693 genes. AlzBase is a collection of genes ranked by frequency of appearance in dysregulated cellular functions in a variety of AD- and AG-related situations. For comparison with gene sets curated in this study "top" genes, called "known AD target genes" here, from the two databases were selected by using frequency thresholds: $>7$ for AlzGene, yielding 106 genes (the top-106 genes), and >15 for AlzBase, yielding 109 genes (the top-109 genes) (Supplementary Table 2).

\section{Computational software}


Microarray data processing and analysis were done in the R environment (http://cran.rproject.org/). Differentially expressed genes (DEGs) were selected using LIMMA [36]. platform Cytoscape (v 3.5.1) [http://www.cytoscape.org/]. Functional enrichment analysis of gene sets were carried out using DAVID [33] (v 6.7).

154

155

156

157

158

159

160

161

162

163

164

165

166

167

168

169

170

171

172

173

174

175

176

177

\section{Quality screening of data and differentially expressed genes (DEGs)}

A flowchart of computational procedures is sketched in Figure 1. Some of the raw dataset had poor test-control separation. In particular, PCA analysis showed the test and control of the original SFG dataset to be substantially unseparated in component 1 (33.79\%) but partially separated in component $2(16.46 \%)$, resulting in a score of zero. Two standard quality control tests, DEG-based two-way hierarchical clustering (G2HC) and principal component analysis (PCA), were applied on the raw datasets to assure good test-control separation and similar dataset size. For each case a reduced, a "good separation" set (Supplementary Table 1) of microarrays was determined by pruning microarrays from the original set until there was perfect separation in both the G2HC (Supplementary Figure 1) and PCA (Supplementary Figure 2) tests. PCA analysis of the reduced SFG dataset showed the test and control to be completely separated in component $1(42.10 \%)$ and partially separated in component $2(9.58 \%)$, resulting in a score of 1.0. Pruned microarrays were excluded from subsequent analyses (see effect of dataset reduction in Discussion). In the ensuing analysis in R environment, non-sense genes and gene duplications were removed from the 46,141 probe sets, leaving 21,765 genes for all cases. Significant genes were then culled according to false-discovery ratio (FDR) of the fold-change (FC) in gene expression. Approximately 800 DEGs per case were selected by LIMMA [36] with the criteria $|\mathrm{FC}(\log 2)|>1$ and case-dependent FDR thresholds (for AG, 7.50E-04; EC, 4.0E-06; HC, 1.0e-05; MTG, 5.0e-06; PC, 1.0e-04; SFG, 1.25e-05).

\section{Selection of differentially co-expressed gene (DCE) pairs}

DCE analysis was done in programming language C. The method of Wang [37] was used to evaluate the difference between test and control in co-expression correlation of gene-pairs, as follows. Separately for test and control samples, gene expressions were normalized over cohort and converted to $t$-scores. The linear regressions, $r$, of the $t$-scores were obtained for every gene- 
178 pair. A positive/negative $r$ implies the gene-pair is positively/negatively correlated

179 (Supplementary Figure 3). A pair is said to have gain of co-expression (GOC) if $r_{\mathrm{t}}>r_{\mathrm{c}}$, and loss 180 of co-expression (LOC) if $r_{\mathrm{t}}<r_{\mathrm{c}}$, where the subscript $\mathrm{t}$ (c) stands for test (control). To select 181 gene-pairs with significant change of co-expression, the linear regression $r$ was converted to a 182 heterogeneity statistic $Q$ [37],

$$
Q=\left(n_{\mathrm{t}}-3\right)\left(z_{\mathrm{t}}-[z]\right)^{2}+\left(n_{\mathrm{c}}-3\right)\left(z_{\mathrm{c}}-[z]\right)^{2},
$$

where $n_{\mathrm{t}}\left(n_{\mathrm{c}}\right)$ is the test (control) cohort size, $z$ is Fisher's $z$-statistics

$$
z=(1 / 2) \log ((1+r) / 1-r)),
$$

and $[z]$ is the $z$ averaged over all pairs. Gene pairs having top- $0.1 \%$ Q-statistics, corresponds to a lower-bound Q-threshold ranging from 4.24 to 5.86 (Supplementary Figure 4), were selected as DCE pairs, yielding approximately 240,000 pairs for each case (Supplementary Table 3).

Construction of networks from interacting differentially co-expressed gene (IDCE) pairs

Case specific interacting gene networks (IGNs) were constructed by integration of DCE pairs with protein-protein interaction (PPI) data: the Human Protein Reference Database (HPRD) [31] and Uniprot [32]. If two genes form a DCE pair and if, according to the PPI data, the pair has PPI, then the two genes form an IDCE pair and, together with the link between them, are included in the IGN. Approximately 300 genes were selected by IGNs for each case. It turned out that in each case the IGNs contained only a tiny portion of the entire set of DCE pairs. To examine the effect of this restriction to our analysis, each of the IGN was expanded to a corresponding extended IGN (xIGN) as follows: Add to the IGN any gene (call it gene G) that has a PPI with at least one of the existing genes in the IGN (call it gene A) and is a DCE partner with at least another existing gene in the IGN (call it gene B), provided genes A and B are connected in the IGN. Then G and B form an IDCE pair in xIGN. By definition A and B cannot be the same gene, otherwise G would have already been in IGN. Whereas an IDCE pair in IGN 202 is a DCE pair with direct PPI, a newly added IDCE pair in XIGN is a DCE pair with once203 removed PPI. Genes in xIGN were also required to have minimum degrees: $>2$ for AG and $>3$ 204 for ALZ.

\section{Functional Profiling of the DEGs and the IGNs}


Genes in each case-specific DEG, IGN, and xIGN were used separately as a gene set for

207

208

209

210

211

212

213

214

215

216

217

218

219

220

221

222

223

224

225

226

227

228

229

230

231

232

233

234

235

236 querying enriched KEGG pathways using DAVID [33] (v 6.7); pathways with $p$-values of Fisher's exact test less than 0.05 were considered significant and ranked by $p$-value.

\section{Results}

\section{Properties of curated gene sets}

Eighteen AG and ALZ gene sets - DEG, IGN, and XIGN sets for AG and five ALZ regions, collectively called curated gene sets, were curated in this work. With case-dependent stringent FDR thresholds about 800 DEGs were selected for each case. The ratio of downregulated to upregulated genes was about 7:3 for AG, about 5:1 for EC, $\mathrm{HC}, \mathrm{PC}$, and $\mathrm{SFG}$, and about 3:4 for MTG (Supplementary Table 4). A two-way clustering of the six cases based on the union of curated DEGs (containing 3,355 genes) put HC, PC, and SFG in a tightly knit group and leaves MTG, EC, and AG as outliers (Supplementary Figure 5). Because of this clustering result and because $\mathrm{HC}$ and $\mathrm{PC}$ are viewed as the most important brain regions characterizing ALZ, in this study we used $\mathrm{HC} / \mathrm{PC} / \mathrm{SFG}$ as the main group for comparison with AG. The number of genes in the interacting gene network (IGN) in each case was approximately 350 . While the average degree per gene (number of links connected to it) was less than 2, a few genes have degrees as high as 14 (Supplementary Table 4. By design the extended interacting gene networks (xIGNs) were much larger than their IGN counterparts; the number of genes was controlled to be approximately 900 by admitting only genes with degree greater than 2 in the case of $A G$, and greater than 3 in the ALZ cases (Supplementary Table 4). The top-10 most significant DEGs in the six cases had FDR values far smaller than their respective FDR thresholds (Supplementary Table 5). Similarly, the highest-degree genes in the IGNs and xIGNs had degrees much higher than the threshold degree (Supplementary Tables 6-7). The overlap between the curated DEG and IGN gene sets was about $10 \%$ of IGN, except for EC (1\%) and HC (24\%). The overlap between IGN and xIGN was about $90 \%$ of IGN, except for PC (40\%), showing that the DEG and IGN sets were substantially distinct, whereas xIGN was basically an enlargement of IGN (Figure 2). In our discussion we focused on IGN and used XIGN only for reference.

\section{Curated gene sets significantly enriched in AlzBase, not AlzGene}

The top-106 AlzGene and top-109 AlzBase gene sets are essentially distinct from each other, having only three genes - PGK1, GAPDH, and CDK5 - in common. The AlzGene set, 
237 collected mainly from SNP experiments, was poorly enriched in the curated genes sets; with few 238 exceptions the enrichment $p$-values were greater than $10^{-2}$. In contrast, the AlzBase set, collected 239 mainly from DEG experiments, was highly enriched in the curated set; with few exceptions the 240 enrichment $p$-values were greater than $10^{-10}$, and in a majority of cases less than $10^{-15}$

241 (Supplementary Table 8, Figure 3). The DEG set having the highest enrichment in the AlzBase 242 set was AG-DEG, with $p$-value=7.5E-64, followed by SFG-DEG (1.9E-44); the set having by far 243 the lowest enrichment was EC-DEG (3.4E-03). These observations are consistent with our two244 way clustering of the six DEG sets (Supplementary Figure 5). The top-5 genes (in frequency of 245 appearance) from AlzGene and AlzBase were distinct from each other. With one exception - the 246 AlzGene gene $A B C A 1$, an up-regulated gene in DEG sets of AG, EC, and MTG that did not 247 appear in any of the curated IGN sets - they all appeared down-regulated genes in one or more of 248 the curated DEG sets and in one or more of the ALZ-IGN sets. None of the top-5

249 AlzGene/AlzBase genes played a significant role in our (later) functional analysis of the curated 250 gene sets (Table1).

251

252

253

254

255

256

257

258

259

260

261

262

263

264

265 266

\section{Five major AD culprit genes were not prominent in the curated gene sets}

The five AD culprit genes, APP, MAPT (or tau protein), APOE, PSEN1, and PSEN2, believed to have key roles in AD-genesis, had DCE partners in the brain regions except EC (Supplementary Table 9). SFG had by far the largest number (80) of culprit related DCE pairs, with $\mathrm{HC}$, at 14 pairs, a remote second. In SFG, MAPT, which codes the tau protein and whose over-expression can result in the self-assembly of axonal tangles, had 41 DCE partners; $A P O E$, associated with the common late onset familial and sporadic forms of $\mathrm{AD}$, had 24; $A P P$, which codes amyloid- $\beta$ protein precursor (A $\beta$ PP) and whose proteolysis leads to the formation of $A \beta$, a primary component found in the brain of AD patients, had 10 (Supplementary Table 9).

In spite of their numerous DCE partners, the culprit genes either did not appear in the curated ALZ gene sets or, if they did, were of low ranking. PSEN1 had no IDCE partners, either in IGN or xIGN. APOE was the only culprit gene that appeared in IGN; it had a single IDCE partner in SFG. The culprit genes had more IDCE partners in the larger xIGNs. Two culprit genes were among the DEGs: PSEN1 in EC (upregulated) and PC (downregulated), and PSEN2 in MTG (downregulated). MAPT and APOE were in both the curated IGN and xIGN sets of AG (Supplementary Table 10).

Peer) reviewing PDF | (2019:08:39955:1:0:NEW 13 Jan 2020) 
267

268

269

270

271

272

273

274

275

276

277

278

279

280

281

282

283

284

285

286

287

288

289

290

291

292

293

294

295

296

297

\section{KEGG pathways were enriched heterogeneously in curated gene sets}

Many KEGG pathways were heterogeneously enriched (Fisher's exact test $p$-values $<0.05$ ) in the curated gene sets. Relative to the size of the curated set, more KEGG pathways were enriched in the IGN sets than in the DEG sets. This probably is because the IGNs were distilled interaction networks; interrelation between genes in the IGNs was more similar to that in the KEGG pathways (Supplementary Table 11). The patterns of KEGG pathways enriched in curated IGN and xIGN sets were broadly similar, but were substantially different from those enriched in curated DEG sets (Supplementary Tables 12-14). In what follows we shall focus on the DEG and IGN sets.

The categories of KEGG pathways enriched in DEG and IGN genes for AG and ALZ regions were substantially different, despite important similarities (Supplementary Tables 1214). Category of pathways both DEG- and IGN-enriched included cell proliferation, neurodegeneration, protein complexes and metabolism, and inflammation. Pathways in the category of metabolic related pathways were all DEG-enriched, but not IGN-enriched, and those in carcinoma, the opposite (Figure 4).

\section{OXPHOS and pathways of the three neurodegenerative diseases highly enriched in DEG} sets of HC, PC, and SFG

The most prominent feature of the KEGG analysis of the DEGs were the extremely high enrichment of (the KEGG pathways) Oxidative phosphorylation (OXPHOS), Parkinson's disease (PD), Hunting's disease (HD) and Alzheimer's disease (AD) in the DEG sets of three regions $\mathrm{HC}, \mathrm{PC}$, and SFG. In addition to these four pathways, Proteasome, Pyruvate metabolism, and TCA cycle were also among the Top-10 enriched KEGG pathways in the three regions, and Vibrio cholerae infection and Pathogenic E. coli infection, in two of the three regions. KEGG enrichment in the other three cases were weaker; in order of descending enrichment significance: AG, MTG, and EC. Top-10 enriched pathways in MTG include OXPHOS, V. cholerae infection, and Pathogenic E. coli infection, and Epithelial cell signaling in H. pylori infection, which it shared with AG and SFG. This last pathway was the only top-10 KEGG pathway AG had common with the ALZ cases. EC, which was identified as an outlier in our earlier comparative study of the curated DEG lists, had only four significantly enriched KEGG pathways, including HD (Figure 4, Supplementary Table 12).

Proteasome was the KEGG pathway by far the most enriched in IGN genes 
298

299

300

301

302

303

304

305

306

307

308

309

310

311

312

313

314

315

316

317

318

319

320

321

322

323

324

325

326

327

Proteasome was by far the most prominent pathways in the KEGG analysis of curated IGN genes; it was the pathway with the highest enrichment significance in five cases including AG, but was not significant in SFG. Pathogenic E. coli infection significant was significant in all six cases. Ribosome, not seen in DEG analysis, was significant in all five ALZ cases. OXPHOS, $\mathrm{AD}, \mathrm{PD}, \mathrm{HD}$ were significant in HC (but less so than in DEG) but not in PC and SFG, nor in MTG. AD and HD was also significant in AG, and HD (weakly) in EC. Five cancer pathways, Pathways in cancer, Endometrial cancer, Prostate cancer, Chronic myeloid leukemia, Colorectal cancer were significant in $\mathrm{AG}$, the last three pathways also in PC, and last pathway in PC. No cancer related pathways were significant in HC, MTG, and EC. (Figure 4, Supplementary Table 13).

\section{DEG and IGN genes enriched in the same KEGG pathway tend to be distinct}

When a KEGG pathway was both DEG- and IGN-enriched in a given case, the enriched genes from the two lists were generally quite different, perhaps not surprising because selection criteria for DEG - significant change in expression level - and IGN - significant change in correlation of expressions of gene pair - were distinct, and because only a small portion of genes are common to both DEG and IGN (Figure 2). Enriched genes present in both IGN lists of AG and ALZ tended to have very different GOC and LOC linkages, and some enriched genes present in multiple curated ALZ gene lists were absent in the corresponding AG lists.

\section{ALZ-only hits and novel target genes for the KEGG AD pathway}

Excluding the three neurodegenerative diseases, pathways that were among at least two of the three top-10 KEGG pathways of HC/PC/SFG were OXPHOS, Proteasome, Pyruvate metabolism, TCA cycle, V. cholerae infection and Pathogenic E. coli infection (Supplementary Table 15-16). Genes in the gene lists of these six pathways that were DEG hits in at least two of $\mathrm{HC} / \mathrm{PC} / \mathrm{SFG}$ but not in AG were collected into a set of 54 "ALZ-only hits", of which 43, 47, and 36, respectively, were DEGs of HC, PC, and SFG; all were down-regulated (Figure 5 and Supplementary Table 16). AlzBase contains two of the hits, ATP5B and ATP5G3, and AlzGene, none. Of the 54 hits, 26 were from OXPHOS, 14 from Proteasome, and 10 from Pyruvate metabolism and TCA cycle combined (one shared with OXPHOS and 4 are common to both), and 5 from E. coli infection. All OXPHOS hits except SDHA (the hit shared with TCA cycle) were mitochondrial enzymes: 9 belong to the ATP family of genes; 9, NDU; 4, COX; and 3, 
328 UQCR. None of the hits not in OXPHOS were mitochondrial. All Proteasome hits except POMP 329 were from the PSM family of enzymes. All the OXPHOS hits except ATP6V1E1, ATP6V1H, $330 A T P 5 J 2$, and $A T P 5 L$ were also in the KEGG AD pathway, and none of the other 32 hits, call 331 "novel AD genes", were (Table 2, Figure 5).

332

\section{Discussion}

\section{Comparison with original analysis of AG dataset}

335 In a comprehensive study, the authors of the AG dataset presented evidence suggesting that 336 the transcription factor REST (also known as neuron-restrictive silencer factor, NRSF) is

337

338

339

340

341

342

343

344

345

346

347

348

349

350

351

352

353

354

355

356

357

358 normally induced and acts as a universal feature of normal ageing in human cortical and hippocampal neurons, and a neuroprotective modulator by repressing genes that promote cell death and the pathology of $\mathrm{AD}$, and confers oxidative stress resistance and protects against toxic insults, such as $\mathrm{A} \beta$ oligomers and tau phosphorylation, associated with $\mathrm{AD}$, but is lost in mild cognitive impairment and $\mathrm{AD}[30]$. In our study the gene REST was moderately up-regulated in AG and, in spite of what was suggested in [30], similarly in all five ALZ datasets, but, with a FC ( $\log 2$ ) ranging from 0.541 (PC) to 0.914 (SFG), was not a DEG (Supplementary Table 17). Our result does not directly contradict [30] because the AD work in [30] was not genomic based. Our ALZ study did yield many strong AD signatures, including a severe dysfunction of OXPHOS.

\section{Comparison with original analyses of ALZ datasets}

The ALZ datasets (we did not analyze the dataset collected from the primary visual cortex) were also analyzed in $[28,29,38]$. In methodology the main difference between the analyses of these authors and us was the reduction of the datasets so that all datasets were of similar size (about 10 vs. 10) and, more importantly, had good test-control separation in PCA analysis. In addition, for function analysis [38] used Gene Ontology (GO) and we used KEGG pathways. In practice our DEG results are quantitatively (Supplementary Table 18) and qualitatively consistent with the results in [38], except for the case of SFG. This is perhaps to be expected when the PCA analysis for the original SFG set (23 control vs. 11 test) had a score of zero, whereas the reduced set (12 vs. 8 ) had a score of 1 . When the screening criteria (FDR $<1.25 \mathrm{E}-$ $05,|F C|>1$ ) used to selected the 829 DEGs from the reduced SFG set were applied to the original set the yield was zero; using the criteria FDR $<1.00 \mathrm{E}-02,|\mathrm{FC}|>1$ yielded 395 DEGs, which had 92 common genes with the 829-gene set (Supplementary Table 18). The functional 
359

360

361

362

363

364

365

366

367

368

369

370

371

372

373

374

375

376

377

378

379

380

381

382

383

384

385

386

387

388

389

analyses also yielded drastically different results for SFG. In our result SFG belonged to the same category as HC and PC, whereas in [38] it was an outlier and shared no common feature with $\mathrm{HC}$ or PC. For instance, mitochondrial functions were reported to be severely disrupted in HC, PC, and MTG but not in SFG [29], whereas our result indicated it to be severely disrupted in all four regions. In both analyses EC was also an outlier, separate from SFG in [38]. We emphasize that concerning SFG there is no contradiction between ours and the analysis in [28, 29, 38]; different sets of microarrays were analyzed, which yielded different presentations of the pathology of SFG, differences which may be resolved by new data and analyses.

\section{Comparison with AlzGene and AlzBase}

That our curated gene set has very low enrichment in the AlzGene set but much higher enrichment in the AlzBase set (Figure 3) could be because gene expression was used to selected genes in AlzBase, as was in the present work, whereas SNP, one of the most common types of genomic variation, was used to selected genes in AlzGene. The AlzBase set was more enriched in our AG than ALZ gene sets because it (AlzBase) was curated from AD brain as well as AG brain [35]. Gene expression as a leading determinant factor may explain why the DEG subsets of the curated genes were, overall, more enriched than the IGN and xIGN subsets in the AlzBase set, which was essentially determined by DEG. The IGN and xIGN subsets were determined by co-expression of gene-pairs as well as PPI data. It is notable that the five major AD culprit genes were not prominent among the curated genes. In comparison, the top-106 AlzGene gene set contains the four culprit genes APOE, MAPT, PSEN1, APP (in descending order of appearance frequency), but the top-109 AlzBase gene set contains none. Culprit genes most likely were more active during the onset of $\mathrm{AD}$ in the patients and less, or even not, active during late stages of AD. In contrast, curated genes were culled from microarray data taken postmortem from patients, whose deaths presumably were caused by advanced states of AD.

\section{Cancer-related pathways disrupted in AG, essentially not in ALZ}

Most of the leading disrupted KEGG pathways in AG were not disrupted in ALZ. Those that were type-1 disrupted were mainly signaling pathways related to cancer: MAPK signaling pathway [39], Cell adhesion molecules [40], and Calcium signaling pathway [41]

(Supplementary Table 12), and type-2 disrupted were pathways related to specific cancers: Pathways in cancer, Endometrial cancer, Prostate cancer, and Colorectal cancer (Supplementary Table 13). Because AG compared old (70 years and above) versus young (40 and below), 
390

391

392

393

394

395

396

397

398

399

400

401

402

403

404

405

406

407

408

409

410

411

412

413

414

415

416

417

418

419

420

whereas ALZ compared AD patients versus age-matched (65 and above) non-AD cohorts, the above results do not imply AD patients did not have cancer-related dysfunctions, rather, it meant such dysfunctions were not exacerbated by $\mathrm{AD}$. It has been suggested that an inverse comorbidity relation exists between $\mathrm{AD}$ and some types of cancer, including smoke-related ones $[42,43]$. Our result does not directly support it.

\section{OXPHOS and Proteasome strongly disrupted in ALZ, not in AG}

Brain related dysfunctions, especially mitochondrial dysfunction, are often discussed in the context of both old age and AD. In this work there was clear distinction between the two conditions. OXPHOS and Proteasome were the two most strongly disrupted pathways in ALZ 40 of the 54 ALZ-only genes were either mitochondrial or proteasomal, yet OXPHOS was not at all disrupted, and Proteasome was only type-2 disrupted, in AG (Supplementary Tables 12 and 13). The Proteasome genes enriched in the IGN-AG gene list were mostly distinct from those enriched in IGN-ALZ list (Table 2). This suggests loss of mitochondrial and/or proteasomal function in the non-AD aged has the potential of being used as an indicator for early onset of AD.

\section{OXPHOS and the three neurodegenerative pathways}

The four KEGG mitochondria related pathways OXPHOS, AD, HD, and PD, together with Proteasome, exhibited by far the strongest type 1 and type 2 disruptions in the ALZ datasets, especially in HC/PC/SFG (a KEGG pathway is type 1 or 2 disrupted when it is enriched in a DEG or IGN set). Of the 133 genes in (the) OXPHOS (gene set) only 6 are not from one of the six families of (genes coding for) mitochondrial enzymes: NADH dehydrogenase (NDH), NADH: ubiquinone oxidoreductase (NDU), succinate dehydrogenase complex (SDH), cytochrome c oxidase (COX), ATP synthase (ATP), ubiquinol-cytochrome c reductase (UQCR). Mitochondrial genes are also preponderant in the gene lists of the three neurodegenerative diseases: 89/171, 95/142, and 89/193, respectively, in AD, PD, and HD. The DEGs of $\mathrm{HC} / \mathrm{PC} / \mathrm{SFG}$ were also rich in mitochondrial genes of OXPHOS, 28, 42, and 32 genes, respectively, in HC, PC, and SFG (Supplementary Table 19). Aside from the AD culprit genes related to $\mathrm{A} \beta$ production, the $68 \mathrm{AD}$-specific genes (not common with OXPHOS or HD or PD, hence also non-mitochondrial) in $\mathrm{AD}$ are dominated by genes related to signal transduction, including genes in the CAC (Calcium Voltage-Gated Channel), CAL (Calmodulin), CAP (Calpain, Calcium-Activated Neutral Proteinase), GRIN (Glutamate Ionotropic Receptor 
421 NMDA), ITPR (Inositol 1,4,5-Trisphosphate Receptor). Only 7 of these 68 AD-specific genes 422 were hits in DEG-HC (6/5 hits in PC/SFG), less than the 8 hits in DEG-AG. In comparison, $4236 / 19 / 14 / 10$ of the HD and/or PD-specific genes were hits in AG/HC/PC/SFG, suggesting that the 424 KEGG AD pathway, as it currently stands, is not particularly AD specific (Supplementary Table 425 19).

\section{OXPHOS dysfunction and tau and $\mathrm{A} \beta$ pathologies}

427 OXPHOS is the metabolic pathway in the mitochondria that produces ATP, whose energy is 428 released to power the brain. Dysfunction of mitochondria has long been associated with AD [4447], PD [48], and HD [49]. Experiments and functional analysis on P301L tau transgenic mice demonstrated mitochondrial dysfunction leads to reduced NDU activity and impaired ATP and the suggestion that tau pathology involves a mitochondrial and oxidative stress disorder possibly distinct from that caused by $\mathrm{A} \beta$ synthesis $[45,50]$. Experiments on triple transgenic $\mathrm{AD}$ mice found a massive deregulation of 24 proteins, including 3 in the NDU family (in mitochondria complex I) and 3 in the COX family (complex IV). Because deregulation of complex I was tau dependent, whereas deregulation of complex IV was $A \beta$ dependent, the authors concluded that OXPHOS was synergistically impaired by $\mathrm{A} \beta$ and tau $[50,51]$. Results in this work suggest wide spread mitochondrial dysfunction; the ALZ-only list includes 9 NDU, 1 SDH (complex II), 4 UQCR (complex III), 4 COX, and 9 ATP (complex V) coding genes (Table 2, Figure 5), all down-regulated; but not any ND (complex 1) coding genes.

440

441

442

443

444

445

446

447

448

449

450

451

\section{The Proteasome and $A \beta$ pathology}

The ubiquitin-proteasome system (UPS) is an important part of the proteolysis machinery in eukaryotic cells; it maintains proteostasis [52], regulates proteins biosynthesis, and controls levels of presynaptic protein [53]. UPS was associated with AD when ubiquitin was detected in neurofibrillary tangles and senile plaques in $\mathrm{AD}$ affected brains [54, 55]. Proteasome is the core of UPS that degrades ubiquitinated proteins. Cell model experiments have shown that the proteasome is inhibited by, but does not directly degrade A $\beta$. Rather, it degrades PS1 and PS2 as well as their mutated forms. Mutated PSEN1/PSEN2 leads to the production of the plaqueforming peptide, $A \beta 42$, and the inhibition of proteasome was shown to enhance this production [56]. In the proteasome, degradation of ubiquitinated proteins/peptides is carried out in the barrel-shaped 20S complex, composed of four types $-\alpha / \beta / \gamma / \delta$ - of proteasome subunits $(P S M A / B / C / D)$. The $\beta$-subunits contain peptide cleaving activities, caspase-like by $P S M B 1 / 6$, 
452 trypsin-like by $P S M B 2 / 4 / 7$, and chymotrypsin-like by $P S M B 5$, whereas the other three type of 453 subunits have structural and regulatory functions. Experiments on autopsied AD affected brains 454 (and controls) recorded a decrease in caspase-like and chymotrypsin-like activities in AD brain, 455 but saw no significant decrease in either $\alpha$ - or $\beta$-subunit expression [57]. However, whereas the 456 authors reported statistical significance for the activity results, the same was not provided for the 457 expression result. The gene $P O M P$ encodes a molecular chaperone that is essential for $20 \mathrm{~S}$ 458 proteasome formation. Its overexpression increases proteasome function and enhances 459 proteasome-mediated antioxidant defense [58]. Strong type 1 and type 2-disruption of 460 Proteasome was one of the most prominent features of our result. In contrast the only ubiquitin 461 related pathway noticeably enriched in our data was hsa04120: Ubiquintin mediated proteolysis, 462 in IGN-EC. The ALZ-only hits from Proteasome include POMP and 13 PSM genes covering all 463 four types of subunits, including six of the seven $\beta$-subunits, PSMB1 to PSMB6; all were down 464 regulated (Table 2, Figure 5). The KEGG pathway hsa04142: Lysosome pathway, system of 465 organelles that digests waste macromolecules autophagy in the cytoplasm, did not surface in our 466 work.

\section{Pyruvate metabolism, TCA cycle, and dementia}

In adult human, the brain accounts for about $2 \%$ of the body weight but consumes about

469

470

471

472

473

474

475

476

477

478

479

480

481

482

$25 \%$ of body glucose. OXPHOS, pyruvate metabolism, and the TCA (Krebs) cycle are key glucose metabolic pathways. Pyruvate, the end product of glycolysis, is converted by the pyruvate dehydrogenase (PDH) complex to acetyl CoA, which is taken by the TCA cycle to produce nicotinamide adenine dinucleotide (NADH), which is fed into the OXPHOS process to produce ATP. Aberrant glucose metabolism is a feature of AD pathology [59]. In addition to $\mathrm{PDH}$, key enzymes in pyruvate metabolism and the TCA cycle include lactatedehydrogenase (LDH), $\alpha$-ketoglutarate dehydrogenase (DLD), isocitrate dehydrogenase (IDH), malate dehydrogenase $(\mathrm{MDH})$, and succinate dehydrogenase (SDH). A reduced glycolytic energy production, such as caused by aberrant pyruvate metabolism, is a common symptom of $\mathrm{AD}$ patients [60]. Decreased PDH and COX activity in female 3xTg-AD mice have been reported [61]. Activities of TCA cycle enzymes in brains from patients with autopsy-confirmed AD and clinical dementia ratings (CDRs) before death have been measured, and significant $(p<0.01)$ decreases in the activities of the PDH complex, IDH, and the DLD complex, and increases in $\mathrm{SDH}$ and MDH were reported [62]. Experiments on prematurely aging mtDNA mutator mice

PeerJ reviewing PDF | (2019:08:39955:1:0:NEW 13 Jan 2020) 
483 suggested that decreased LDHB (relative to LDHA) leads to CNS aging in mice [63]. In this 484 work both Pyruvate metabolism and TCA cycle pathways were type 1 disrupted in $\mathrm{HC} / \mathrm{PC} / \mathrm{SFG}$ $485 \quad(p$-value $=2.9 \mathrm{E}-04 / 6.1 \mathrm{E}-03 / 3.4 \mathrm{E}-03$ for Pyruvate; $1.4 \mathrm{E}-03 / 2.7 \mathrm{E}-02 / 1.4 \mathrm{E}-05$ for TCA $)$, and 486 neither was in AG. Seven enzyme coding genes associated with the two pathways, including 487 those encoding $L D H B, D L D, M D H 2$, PDHA1, $P D H B$, were among the ALZ-only genes; all were 488 down-regulated (Table 2, Figure 5).

\section{Pathogenic E. coli infection, tubulin, and tau tangle}

490

491

492

493

494

495

496

497

498

499

500

501

502

503

504

505

506

507

508

509

510

511

512

513

Microtubules are dynamic structures that form part of the cytoskeleton and are composed of heterodimers of $\alpha$ - (TUBA) and $\beta$-tubulin (TUBB). The aggregation of the microtubuleassociated protein tau in the form of tangles in the brain is one of the two (the other being aggregation of $\mathrm{A} \beta$ plaques) manifests of $\mathrm{AD}[64]$. Bacterial pathogens including E. coli (and $V$. cholerae) induce microtubule destruction in their invasion of host [65]. Level of E. coli $\mathrm{K} 99$ was reported to be greater in AD compared to control brains [66]. Loss of microtubule [67] and tubulin [68] have been reported in the neurons of AD brains. Level of reduced $\alpha$-tubulin expression led to increased human-tau expression in transgenic worm [69]. In this work the KEGG pathway Pathogenic $E$. coli infection was type 1 disrupted in $\mathrm{HC} / \mathrm{PC} / \mathrm{SFG}$ ( $p$-value = 3.9E-02/1.1E-03/7.2E-03) and MTG (7.2E-03), as well as in AG (6.7E-03), and type 2 disrupted in all six cases. The ALZ-only set had five genes associated with the pathway, including two $\alpha$ tubulin genes TUBA1B and TUBAIC and two $\beta$-tubulin genes TUBB and TUBB3; all were downregulated (Table 2, Figure 5).

$V$. cholerae is gram-negative bacterium known for causing the disease cholera. The KEGG pathway $V$. cholerae infection was type 1 disrupted in $\mathrm{HC}, \mathrm{SFG}, \mathrm{MTG}$ as well as in AG. Of this KEGG pathway, the 14 ATPase-coding hits in AG and/or ALZ were also OXPHOS hits in AG/ALZ, two - ATP6VIE1 and ATP6V1H - were ALZ-only; both are already in the gene list of the AD pathway. There were a few non-ATPase-coding hits but none satisfied ALZ-only criteria. No literature was found specifically linking $V$. cholerae to AD.

\section{Antimicrobial Protection Hypothesis of AD}

10 Recently it has been shown, based on experiments in which A $\beta$-expressing, APP knockout, and mouse models, as well as worm and mammalian cell models were infected with pathogens including $C$. albicans and $S$. Typhimurium, that $\mathrm{A} \beta$ may be a natural antibiotic that protects the brain from bacterial infection through the adhesion to and agglutination of invading microbes by 
514 oligomerized and fibrillized $\mathrm{A} \beta$ [70]. This work led to the proposal of a new AD

515 amyloidogenesis model, in which $A \beta$ deposition is an early innate immune response to microbial 516 invasion, during which $\mathrm{A} \beta$ first entraps and neutralizes invading pathogens, then fibrillizes and

517 drives neuroinflammatory pathways that help fight the infection and clear $A \beta$ and pathogen

518 deposits. Chronic activation of these pathway leads to sustained inflammation and AD [71]. The 519 new model, called the Antimicrobial Protection Hypothesis of AD by its authors, retains the A $\beta$ 520 production mechanism of the Amyloid Cascade Hypothesis. Implicit in the new hypothesis is 521 AD tends to be associated with old age because the mechanism for $\mathrm{A} \beta$ clearance gradually 522 breaks down with age. The present work is consistent with the new hypothesis in that three 523 pathogens infection related KEGG pathways showed disruption in our datasets: Epithelial cell 524 signaling in Helicobacter pylori infection, type 1 in AG, MTG, and SFG; Pathogenic E. coli 525 infection, type 1 in all six cases except EC, and type 2 in all six cases; Vibrio cholerae infection, 526 type 1 in AG, HC, MTG, and SFG (Figure 4, Supplementary Table 12). Recently, experiments in 527 transgenic mouse model have shown that acute treatment of $A \beta$ induces microglia, brain-resident 528 innate immune cells, activation accompanied by metabolic reprogramming from OXPHOS to 529 glycolysis [72]. The KEGG pathway hsa00010: Glycolysis/Gluconeogenesis was not enriched in 530 any of our curated DEG sets, but was mildly enriched in IGN-HC; no KEGG pathway related to 531 microglia or inflammation surfaced in our work.

\section{Novel genes for the KEGG AD pathway}

533 It is curious that $\mathrm{AD}$ was less type 1-disrupted than $\mathrm{HD}$ and $\mathrm{PD}$ in each of $\mathrm{HC} / \mathrm{PC} / \mathrm{SFG}$ 534 (Supplementary Table 12). Twenty-two of the 54 ALZ-only genes were already in the KEGG 535 AD pathway gene set (Table 2). This set included a single non-mitochondrial gene, SDHA; the 536 others were all mitochondrial from OXPHOS. Of the remaining 32 genes, or novel AD genes, 4 537 were ATP genes from OXPHOS, 14 were from Proteasome, and 14 from E. coli, V. cholerea, 538 Pyruvate, and TCA; the 28 non-OXPHOS genes include all the non-mitochondrial genes except $539 S D H A$ in the ALZ-only set (Table 2). In comparison to these 28 non-mitochondrial genes, there 540 were only 8 non-mitochondrial AD pathway hits - SDHA, ATF6, BACE1, CALM1, GAPDH, 541 GSK3B, NAE1, and RTN3 - (Supplementary Table 19) in the ALZ-DEG datasets. In other words, 542 as far as non-mitochondrial genes are concerned the KEGG AD pathway and our ALZ DEG sets 543 are complementary; the former is rich in signal-transduction related genes but lack genes from $E$. 544 coli, V. cholerea, Pyruvate, TCA, Proteasome, whereas the latter is vice versa. When the 32 
545 novel AD genes were added to the KEGG AD pathway set, then, as expected, AD became more

546 type 1- and type 2-disrupted than HD and PD in all ALZ datasets (Figure 6, Supplementary

547 Table 20).

548

549

550

In summary, our analysis revealed strong heterogeneity in AD signatures among the five brain regions; $\mathrm{HC} / \mathrm{PC} / \mathrm{SFG}$ showed clear and pronounced AD signatures, MTG moderately so,

551

552

553

554

555

556

557

558

559

560

561

562

563

564

565

566

567

568

569

570

571

572

573

574 and EC showed essentially none. There were stark differences between ALZ and AG. Whereas OXPHOS and Proteasome were the most disrupted pathways in $\mathrm{HC} / \mathrm{PC} / \mathrm{SFG}, \mathrm{AG}$ showed no OXPHOS disruption and only type 2 Proteasome disruption in AG. Metabolic related pathways including TCA cycle and Pyruvate metabolism were disrupted in ALZ but not in AG. Our result is consistent with the notion that OXPHOS dysfunction is closely related to $A \beta$ and tau pathologies and with the Antimicrobial Protection Hypothesis of AD in that three pathogens infection related pathways were disrupted in ALZ. Many cancer and signaling pathways were disrupted in $\mathrm{AG}$ but our results suggest that having AD does not exacerbate such disorders in the aged. We identified 54 “ALZ-only” differentially expressed genes, all down regulated and which, when used to augment the gene list of the KEGG AD pathway, made it significantly more AD-specific. Because OXPHOS dysfunction is directly tied to mitochondrial and oxidative stress disorder and energy supply to brain cells, our result suggests the potential of monitoring the level of neuron activity as a non-invasive method for detecting the possible early onset of AD in the aged, and that maintaining healthy mitochondria and proteasome may be a worthwhile strategy for preventing the onset, or slowing or arresting the progress of AD.

\section{Limitations and Perspectives}

The general applicability of our results to all AG and AD cases is limited by the relatively small number of microarray samples used in the present study. Future larger-scaled studies will determine whether our decision to analyze subsets of the original AG and AD datasets with better test-control separations was a wise one. Even though our results are generally consistent with experiments reported in the literature (which we cited), it still lacks direct experimental support. Hence our results should best be viewed as possible topics for experimental study and verification, chief among which is the clear AG-AD difference with respect to mitochondria and proteasome dysfunction. We may collaborate with experimental researchers in initiating related 
575 verification projects. We are interested in joining the Oskar Fischer Project and have AD related 576 discussion with other researchers.

\section{Abbreviations}

578 AD/ALZ: Alzheimer's disease; AD for generic use, ALZ when specifically referring to the AD 579 datasets in this study

580 AG: aging

581 IGN: interacting gene network xIGN: extended IGN

582 DCE: differentially co-expressed

583 DEG: differentially expressed genes

584 EC: entorhinal cortex

585 GEO: Gene Expression Omnibus

586 GOC: gain of co-expression

587 HC: hippocampus

588 HD: Huntington's disease

589 HPRD: Human Protein Reference

590 IDCE pair: interacting DCE pair

591 IIP: innate immune pathway

592 KEGG: Kyoto Encyclopedia of Genes and Genomes

593 LOC: loss of co-expression

594 MTG: medial temporal gyrus

595 PC: posterior cingulate

596 PD: Parkinson's disease

597 PPI: protein-protein interaction

598 SFG: superior frontal gyrus

599 Type 1 disruption (of function): function represented by a KEGG pathway is enriched in DEGs

600 Type 2 disruption (of function): function represented by a KEGG pathway is enriched in IGN 601 genes

602

603

604 
605 Reference

606 [1] López-Otín C, Blasco MA, Partridge L, Serrano M, Kroemer G (2013) The hallmarks of

607

608

609

610

611

612

613

614

615

616

617

618

619

620

621

622

623

624

625

626

627

628

629

630

631

632

633

634

635

636

637

638

639

640

641

642

643

644

645

646

647

648

649

650

[7] Selkoe DJ, Hardy J (2016) The amyloid hypothesis of Alzheimer's disease at 25 years. EMBO molecular medicine 8, 595-608.

[8] De Marco M, Vallelunga A, Meneghello F, Varma S, Frangi A, Venneri A, Alzheimers DNI (2017) ApoE $\varepsilon 4$ allele relateted alterations in hippocampal connectivity in early Alzheimer's disease support memory performance. Current Alzheimer research.

[9] Hyman B (2016) Tau and Alzheimer pathobiology. Neurobiology of Aging 39, S16.

[10] Bancher C, Brunner C, Lassmann H, Budka H, Jellinger K, Wiche G, Seitelberger F, Grundke-Iqbal I, Iqbal K, Wisniewski H (1989) Accumulation of abnormally phosphorylated $\tau$ precedes the formation of neurofibrillary tangles in Alzheimer's disease. Brain research 477, 90-99.

[11] Bateman RJ, Siemers ER, Mawuenyega KG, Wen G, Browning KR, Sigurdson WC, Yarasheski KE, Friedrich SW, DeMattos RB, May PC (2009) A $\gamma$-secretase inhibitor decreases amyloid- $\beta$ production in the central nervous system. Annals of Neurology: Official Journal of the American Neurological Association and the Child Neurology Society 66, 48-54.

[12] Timmers M, Streffer JR, Russu A, Tominaga Y, Shimizu H, Shiraishi A, Tatikola K, Smekens P, Börjesson-Hanson A, Andreasen N (2018) Pharmacodynamics of atabecestat (JNJ-54861911), an oral BACE1 inhibitor in patients with early Alzheimer's disease: randomized, double-blind, placebo-controlled study. Alzheimer's research \& therapy $\mathbf{1 0}$, 85.

[13] Egan MF, Kost J, Tariot PN, Aisen PS, Cummings JL, Vellas B, Sur C, Mukai Y, Voss T, Furtek C (2018) Randomized trial of verubecestat for mild-to-moderate Alzheimer's disease. New England Journal of Medicine 378, 1691-1703.

[14] Sevigny J, Chiao P, Bussière T, Weinreb PH, Williams L, Maier M, Dunstan R, Salloway $\mathrm{S}$, Chen T, Ling Y (2016) The antibody aducanumab reduces A $\beta$ plaques in Alzheimer's disease. Nature 537, 50.

[15] Tayeb HO, Murray ED, Price BH, Tarazi FI (2013) Bapineuzumab and solanezumab for Alzheimer's disease: is the 'amyloid cascade hypothesis' still alive? Expert opinion on biological therapy 13, 1075-1084.

[16] Blaettler T, Smith J, Smith J, Paul R, Asnaghi V, Fuji R, Quartino A, Honigberg L, Rabbia MA, Yule S (2016) Clinical trial design of cread: A randomized, double-blind, 
651

652

653

654

655

656

657

658

659

660

661

662

663

664

665

666

667

668

669

670

671

672

673

674

675

676

677

678

679

680

681

682

683

684

685

686

687

688

689

690

691

692

693

694

695

696

placebo-controlled, parallel-group phase 3 study to evaluate crenezumab treatment in patients with prodromal-to-mild Alzheimer's disease. Alzheimer's \& Dementia: The Journal of the Alzheimer's Association 12, P609.

[17] Mullard A (2017) Alzheimer amyloid hypothesis lives on. Nature Reviews Drug Discovery 16, 3-5.

[18] Berchtold NC, Coleman PD, Cribbs DH, Rogers J, Gillen DL, Cotman CW (2013) Synaptic genes are extensively downregulated across multiple brain regions in normal human aging and Alzheimer's disease. Neurobiology of aging 34, 1653-1661.

[19] Wang X, Michaelis ML, Michaelis EK (2010) Functional genomics of brain aging and Alzheimer's disease: focus on selective neuronal vulnerability. Current genomics 11, 618.

[20] Saetre P, Jazin E, Emilsson L (2011) Age-related changes in gene expression are accelerated in Alzheimer's disease. Synapse 65, 971-974.

[21] Chu G, Narasimhan B, Tibshirani R, Tusher V (2002) SAM, "Significance Analysis of Microarrays": Users Guide and Technical Document. Stanford University.

[22] Avramopoulos D, Szymanski M, Wang R, Bassett S (2011) Gene expression reveals overlap between normal aging and Alzheimer's disease genes. Neurobiology of aging 32, 2319. e2327-2319. e2334.

[23] Swerdlow RH (2011) Brain aging, Alzheimer's disease, and mitochondria. Biochimica et Biophysica Acta (BBA)-Molecular Basis of Disease 1812, 1630-1639.

[24] Sekar S, McDonald J, Cuyugan L, Aldrich J, Kurdoglu A, Adkins J, Serrano G, Beach TG, Craig DW, Valla J (2015) Alzheimer's disease is associated with altered expression of genes involved in immune response and mitochondrial processes in astrocytes. Neurobiology of aging 36, 583-591.

[25] Hong L, Huang H-C, Jiang Z-F (2014) Relationship between amyloid-beta and the ubiquitin-proteasome system in Alzheimer's disease. Neurological research 36, 276-282.

[26] Amar D, Safer H, Shamir R (2013) Dissection of regulatory networks that are altered in disease via differential co-expression. PLoS Comput Biol 9, e1002955.

[27] Barrett T, Troup DB, Wilhite SE, Ledoux P, Evangelista C, Kim IF, Tomashevsky M, Marshall KA, Phillippy KH, Sherman PM (2011) NCBI GEO: archive for functional genomics data sets-10 years on. Nucleic acids research 39, D1005-D1010.

[28] Liang WS, Dunckley T, Beach TG, Grover A, Mastroeni D, Walker DG, Caselli RJ, Kukull WA, McKeel D, Morris JC (2007) Gene expression profiles in anatomically and functionally distinct regions of the normal aged human brain. Physiological genomics 28, 311-322.

[29] Liang WS, Reiman EM, Valla J, Dunckley T, Beach TG, Grover A, Niedzielko TL, Schneider LE, Mastroeni D, Caselli R (2008) Alzheimer's disease is associated with reduced expression of energy metabolism genes in posterior cingulate neurons.

Proceedings of the National Academy of Sciences 105, 4441-4446.

[30] Lu T, Aron L, Zullo J, Pan Y, Kim H, Chen Y, Yang T-H, Kim H-M, Drake D, Liu XS (2014) REST and stress resistance in ageing and Alzheimer/'s disease. Nature 507, 448454.

[31] Prasad TK, Goel R, Kandasamy K, Keerthikumar S, Kumar S, Mathivanan S, Telikicherla D, Raju R, Shafreen B, Venugopal A (2009) Human protein reference database-2009 update. Nucleic acids research 37, D767-D772.

[32] Consortium U (2014) Activities at the universal protein resource (UniProt). Nucleic acids

Peer) reviewing PDF | (2019:08:39955:1:0:NEW 13 Jan 2020) 
697

698

699

700

701

702

703

704

705

706

707

708

709

710

711

712

713

714

715

716

717

718

719

720

721

722

723

724

725

726

727

728

729

730

731

732

733

734

735

736

737

738

739

740

741

742

research $\mathbf{4 2}, 7486$.

[33] Da Wei Huang BTS, Lempicki RA (2008) Systematic and integrative analysis of large gene lists using DAVID bioinformatics resources. Nature protocols 4, 44-57.

[34] Bertram L, McQueen MB, Mullin K, Blacker D, Tanzi RE (2007) Systematic metaanalyses of Alzheimer disease genetic association studies: the AlzGene database. Nature genetics 39, 17-23.

[35] Bai Z, Han G, Xie B, Wang J, Song F, Peng X, Lei H (2016) AlzBase: an integrative database for gene dysregulation in Alzheimer's disease. Molecular neurobiology 53, 310319.

[36] Ritchie ME, Phipson B, Wu D, Hu Y, Law CW, Shi W, Smyth GK (2015) limma powers differential expression analyses for RNA-sequencing and microarray studies. Nucleic acids research, gkv007.

[37] Wang K, Narayanan M, Zhong H, Tompa M, Schadt EE, Zhu J (2009) Meta-analysis of inter-species liver co-expression networks elucidates traits associated with common human diseases. PLoS Comput Biol 5, e1000616.

[38] Liang WS, Dunckley T, Beach TG, Grover A, Mastroeni D, Ramsey K, Caselli RJ, Kukull WA, McKeel D, Morris JC (2008) Altered neuronal gene expression in brain regions differentially affected by Alzheimer's disease: a reference data set. Physiological genomics 33, 240-256.

[39] Dhillon AS, Hagan S, Rath O, Kolch W (2007) MAP kinase signalling pathways in cancer. Oncogene 26, 3279.

[40] Bendas G, Borsig L (2012) Cancer cell adhesion and metastasis: selectins, integrins, and the inhibitory potential of heparins. International journal of cell biology 2012.

[41] Monteith GR, Prevarskaya N, Roberts-Thomson SJ (2017) The calcium-cancer signalling nexus. Nature Reviews Cancer 17, 367.

[42] Driver JA, Beiser A, Au R, Kreger BE, Splansky GL, Kurth T, Kiel DP, Lu KP, Seshadri S, Wolf PA (2012) Inverse association between cancer and Alzheimer's disease: results from the Framingham Heart Study. Bmj 344, e1442.

[43] Musicco M, Adorni F, Di Santo S, Prinelli F, Pettenati C, Caltagirone C, Palmer K, Russo A (2013) Inverse occurrence of cancer and Alzheimer disease A population-based incidence study. Neurology 81, 322-328.

[44] Shoffner JM (1997) Oxidative phosphorylation defects and Alzheimer's disease. Neurogenetics 1, 13-19.

[45] David DC, Hauptmann S, Scherping I, Schuessel K, Keil U, Rizzu P, Ravid R, Dröse S, Brandt U, Müller WE (2005) Proteomic and functional analyses reveal a mitochondrial dysfunction in P301L tau transgenic mice. Journal of Biological Chemistry 280, 2380223814.

[46] Phillips NR, Simpkins JW, Roby RK (2014) Mitochondrial DNA deletions in Alzheimer's brains: a review. Alzheimer's \& Dementia 10, 393-400.

[47] Beal MF (2002) Mitochondria in neurodegeneration In Mitochondrial Disorders Springer, pp. 17-35.

[48] Winklhofer KF, Haass C (2010) Mitochondrial dysfunction in Parkinson's disease. Biochimica et Biophysica Acta (BBA)-Molecular Basis of Disease 1802, 29-44.

[49] Damiano M, Galvan L, Déglon N, Brouillet E (2010) Mitochondria in Huntington's disease. Biochimica Et Biophysica Acta (BBA)-Molecular Basis of Disease 1802, 52-61.

[50] Eckert A, Schulz KL, Rhein V, Götz J (2010) Convergence of amyloid- $\beta$ and tau 
743

744

745

746

747

748

749

750

751

752

753

754

755

756

757

758

759

760

761

762

763

764

765

766

767

768

769

770

771

772

773

774

775

776

777

778

779

780

781

782

783

784

785

786

787

788 pathologies on mitochondria in vivo. Molecular neurobiology 41, 107-114.

[51] Rhein V, Song X, Wiesner A, Ittner LM, Baysang G, Meier F, Ozmen L, Bluethmann H, Dröse S, Brandt U, Savaskan E, Czech C, Götz J, Eckert A (2009) Amyloid- $\beta$ and tau synergistically impair the oxidative phosphorylation system in triple transgenic

Alzheimer's disease mice. Proceedings of the National Academy of Sciences 106, 2005720062.

[52] Lecker SH, Goldberg AL, Mitch WE (2006) Protein degradation by the ubiquitinproteasome pathway in normal and disease states. Journal of the American Society of Nephrology 17, 1807-1819.

[53] Speese SD, Trotta N, Rodesch CK, Aravamudan B, Broadie K (2003) The ubiquitin proteasome system acutely regulates presynaptic protein turnover and synaptic efficacy. Current Biology 13, 899-910.

[54] Perry G, Friedman R, Shaw G, Chau V (1987) Ubiquitin is detected in neurofibrillary tangles and senile plaque neurites of Alzheimer disease brains. Proceedings of the National Academy of Sciences 84, 3033-3036.

[55] Mori H, Kondo J, Ihara Y (1987) Ubiquitin is a component of paired helical filaments in Alzheimer's disease. Science 235, 1641-1644.

[56] Checler F, da Costa CA, Ancolio K, Chevallier N, Lopez-Perez E, Marambaud P (2000) Role of the proteasome in Alzheimer's disease. Biochimica et Biophysica Acta (BBA)Molecular Basis of Disease 1502, 133-138.

[57] Keller JN, Hanni KB, Markesbery WR (2000) Impaired proteasome function in Alzheimer's disease. Journal of neurochemistry 75, 436-439.

[58] Chondrogianni N, Gonos ES (2007) Overexpression of hUMP1/POMP proteasome accessory protein enhances proteasome-mediated antioxidant defence. Experimental gerontology 42, 899-903.

[59] Chen Z, Zhong C (2013) Decoding Alzheimer's disease from perturbed cerebral glucose metabolism: implications for diagnostic and therapeutic strategies. Progress in neurobiology 108, 21-43.

[60] Gray LR, Tompkins SC, Taylor EB (2014) Regulation of pyruvate metabolism and human disease. Cellular and molecular life sciences 71, 2577-2604.

[61] Yao J, Irwin RW, Zhao L, Nilsen J, Hamilton RT, Brinton RD (2009) Mitochondrial bioenergetic deficit precedes Alzheimer's pathology in female mouse model of Alzheimer's disease. Proceedings of the National Academy of Sciences 106, 1467014675.

[62] Bubber P, Haroutunian V, Fisch G, Blass JP, Gibson GE (2005) Mitochondrial abnormalities in Alzheimer brain: mechanistic implications. Annals of Neurology: Official Journal of the American Neurological Association and the Child Neurology Society 57, 695-703.

[63] Ross JM, Öberg J, Brené S, Coppotelli G, Terzioglu M, Pernold K, Goiny M, Sitnikov R, Kehr J, Trifunovic A (2010) High brain lactate is a hallmark of aging and caused by a shift in the lactate dehydrogenase A/B ratio. Proceedings of the National Academy of Sciences 107, 20087-20092.

[64] KoSIK KS, Joachim CL, Selkoe DJ (1986) Microtubule-associated protein tau (tau) is a major antigenic component of paired helical filaments in Alzheimer disease. Proceedings of the National Academy of Sciences 83, 4044-4048.

[65] Radhakrishnan GK, Splitter GA (2012) Modulation of host microtubule dynamics by 
789

790

791

792

793

794

795

796

797

798

799

800

801

802

803

804

805

806

807

808

809

810

811

812

813

814

815

816

817

818

819

820

821

822

823

824

825

826

827

828

829

830

831

832

833

834

pathogenic bacteria. Biomolecular concepts 3, 571-580.

[66] Zhan X, Stamova B, Jin L-W, DeCarli C, Phinney B, Sharp FR (2016) Gram-negative bacterial molecules associate with Alzheimer disease pathology. Neurology 87, 23242332.

[67] Cash AD, Aliev G, Siedlak SL, Nunomura A, Fujioka H, Zhu X, Raina AK, Vinters HV, Tabaton M, Johnson AB (2003) Microtubule reduction in Alzheimer's disease and aging is independent of $\tau$ filament formation. The American journal of pathology 162, 16231627.

[68] Zhang F, Su B, Wang C, Siedlak SL, Mondragon-Rodriguez S, Lee H-g, Wang X, Perry $\mathrm{G}, \mathrm{Zhu}$ X (2015) Posttranslational modifications of $\alpha$-tubulin in alzheimer disease. Translational neurodegeneration 4, 9.

[69] Miyasaka T, Shinzaki Y, Yoshimura S, Yoshina S, Kage-Nakadai E, Mitani S, Ihara Y (2018) Imbalanced expression of tau and tubulin induces neuronal dysfunction in $\mathrm{C}$. elegans models of tauopathy. Frontiers in neuroscience 12, 415.

[70] Kumar DKV, Choi SH, Washicosky KJ, Eimer WA, Tucker S, Ghofrani J, Lefkowitz A, McColl G, Goldstein LE, Tanzi RE (2016) Amyloid- $\beta$ peptide protects against microbial infection in mouse and worm models of Alzheimer's disease. Science translational medicine 8, 340ra372-340ra372.

[71] Moir RD, Lathe R, Tanzi RE (2018) The antimicrobial protection hypothesis of Alzheimer's disease. Alzheimer's \& Dementia 14, 1602-1614.

[72] Baik SH, Kang S, Lee W, Choi H, Chung S, Kim J-I, Mook-Jung I (2019) A Breakdown in Metabolic Reprogramming Causes Microglia Dysfunction in Alzheimer's Disease. Cell Metabolism.

[73] Hou C, Wang Y, Liu J, Wang C, Long J (2017) Neurodegenerative disease related proteins have negative effects on SNARE-mediated membrane fusion in pathological confirmation. Frontiers in molecular neuroscience 10, 66.

[74] Okamoto M, Gray JD, Larson CS, Kazim SF, Soya H, McEwen BS, Pereira AC (2018) Riluzole reduces amyloid beta pathology, improves memory, and restores gene expression changes in a transgenic mouse model of early-onset Alzheimer's disease. Translational psychiatry $\mathbf{8}$.

[75] Sonntag K-C, Ryu W-I, Amirault KM, Healy RA, Siegel AJ, McPhie DL, Forester B, Cohen BM (2017) Late-onset Alzheimer's disease is associated with inherent changes in bioenergetics profiles. Scientific reports 7, 14038.

[76] Xu J, Patassini S, Rustogi N, Riba-Garcia I, Hale BD, Phillips AM, Waldvogel H, Haines R, Bradbury P, Stevens A (2019) Regional protein expression in human Alzheimer's brain correlates with disease severity. Communications biology 2, 43.

[77] LABUDOVA O, KITZMUELLER E, Hermann R, CAIRNS N, LUBEC G (1999) Increased phosphoglycerate kinase in the brains of patients with Down's syndrome but not with Alzheimer's disease. Clinical Science 96, 279-285.

[78] El Kadmiri N, Slassi I, El Moutawakil B, Nadifi S, Tadevosyan A, Hachem A, Soukri A (2014) Glyceraldehyde-3-phosphate dehydrogenase (GAPDH) and Alzheimer's disease. Pathologie Biologie 62, 333-336.

[79] Li X, Zhou J, Chen H, Wang F, Mei Q, Sun H (2017).

[80] Nordestgaard LT, Tybjærg-Hansen A, Nordestgaard BG, Frikke-Schmidt R (2015) Lossof-function mutation in ABCA1 and risk of Alzheimer's disease and cerebrovascular disease. Alzheimer's \& Dementia 11, 1430-1438. 
835 [81] Liu S-L, Wang C, Jiang T, Tan L, Xing A, Yu J-T (2016) The role of Cdk5 in

836

837

838

839

840

841 Alzheimer's disease. Molecular neurobiology 53, 4328-4342.

[82] Llorens-Marítin M, Jurado J, Hernández F, Ávila J (2014) GSK-3 $\beta$, a pivotal kinase in Alzheimer disease. Frontiers in molecular neuroscience 7, 46.

[83] Keller J, Huang F, Markesbery W (2000) Decreased levels of proteasome activity and proteasome expression in aging spinal cord. Neuroscience 98, 149-156. 


\section{Figure 1}

Flowchart

Microarray whole-genome gene expression datasets on aging (AG) and Alzheimer's disease cohorts (ALZ) were collected and quality-control screened; significant genes were curated using two independent methods, DEG and IDCE; six curated gene sets were compared with known $A D$ target genes and were used to query the KEGG pathway database to identify putative disrupted biological functions in AG and ALZ; differences in disruptions in AG and ALZ were identified at the gene level to identify novel AD target genes. Results on pathway analysis were discussed and compared with literature, and inferences drawn.

\section{Raw genetic data | Quality control |Curation of significant genes}

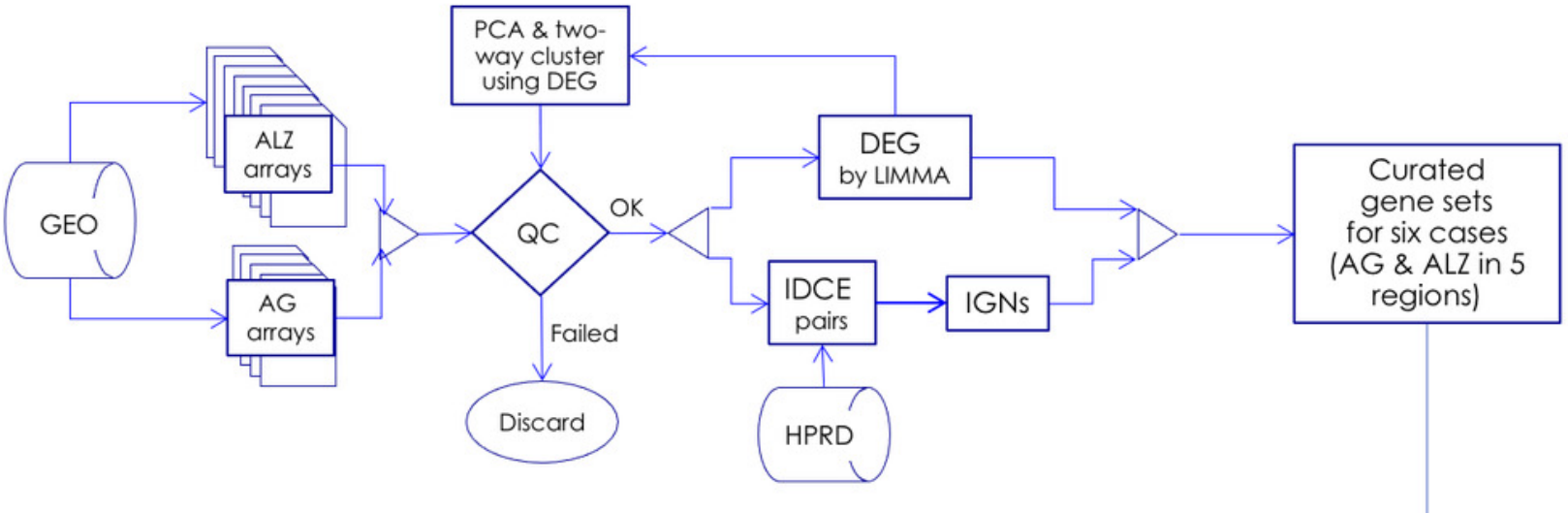

| Check known targets |KEGG analysis | AG-ALZ molecular differences

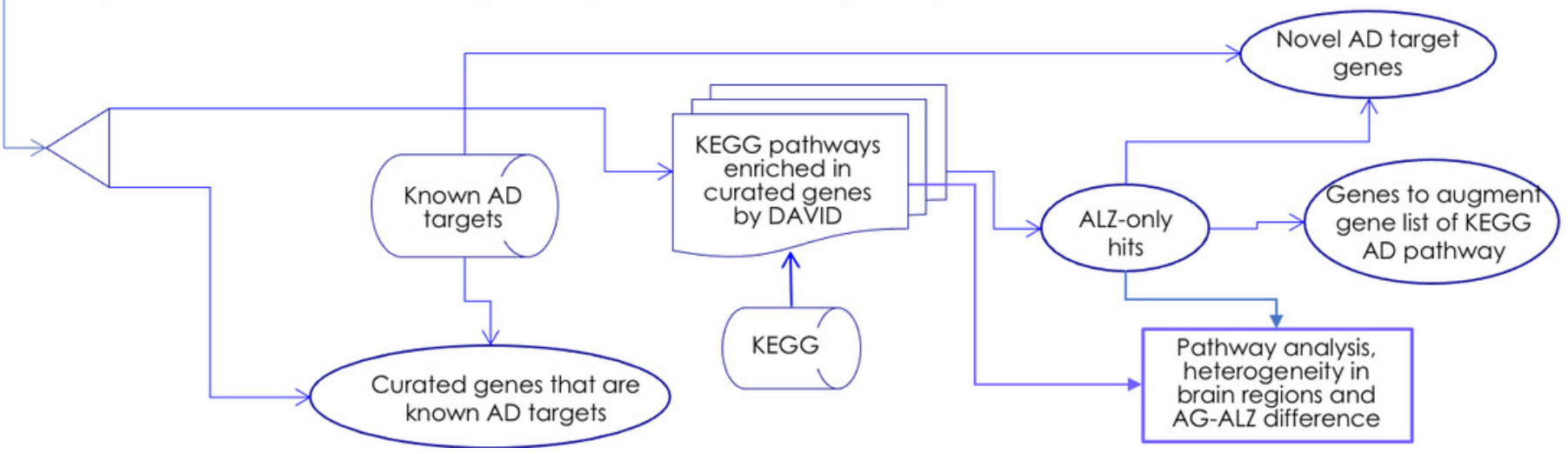


Figure 2

Venn diagrams for six sets of DEG, IGN, and XIGN

Complete lists of all curated DEG (differentially expressed gene), IGN (interacting gene network), and XIGN (extended IGN) gene sets for the six cases may be accessed at the Figshare database link: https://figshare.com/s/246b773e7c1dad2b26a7 . Abbreviation: AG, normal aging; EC, entorhinal cortex; HC, hippocampus; MTG, medial temporal gyrus; PC, posterior cingulate; SFG, superior frontal gyrus. 
(AG)

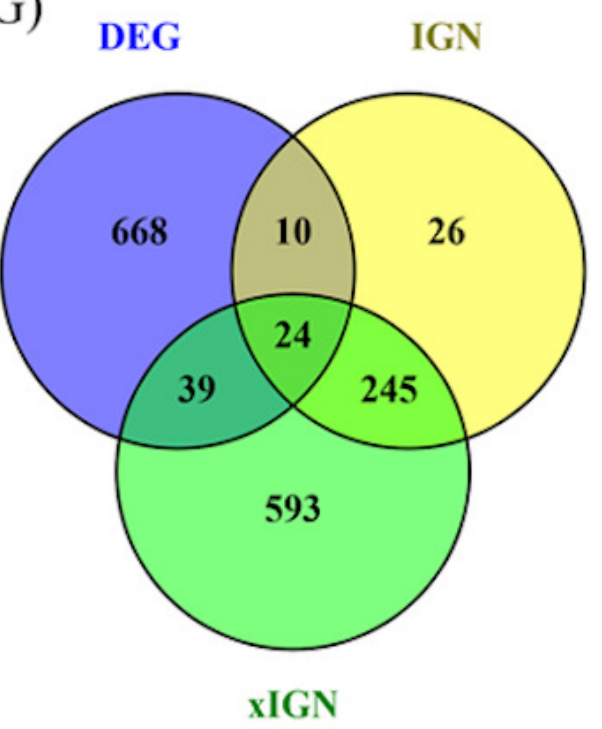

( $\mathrm{HC})$
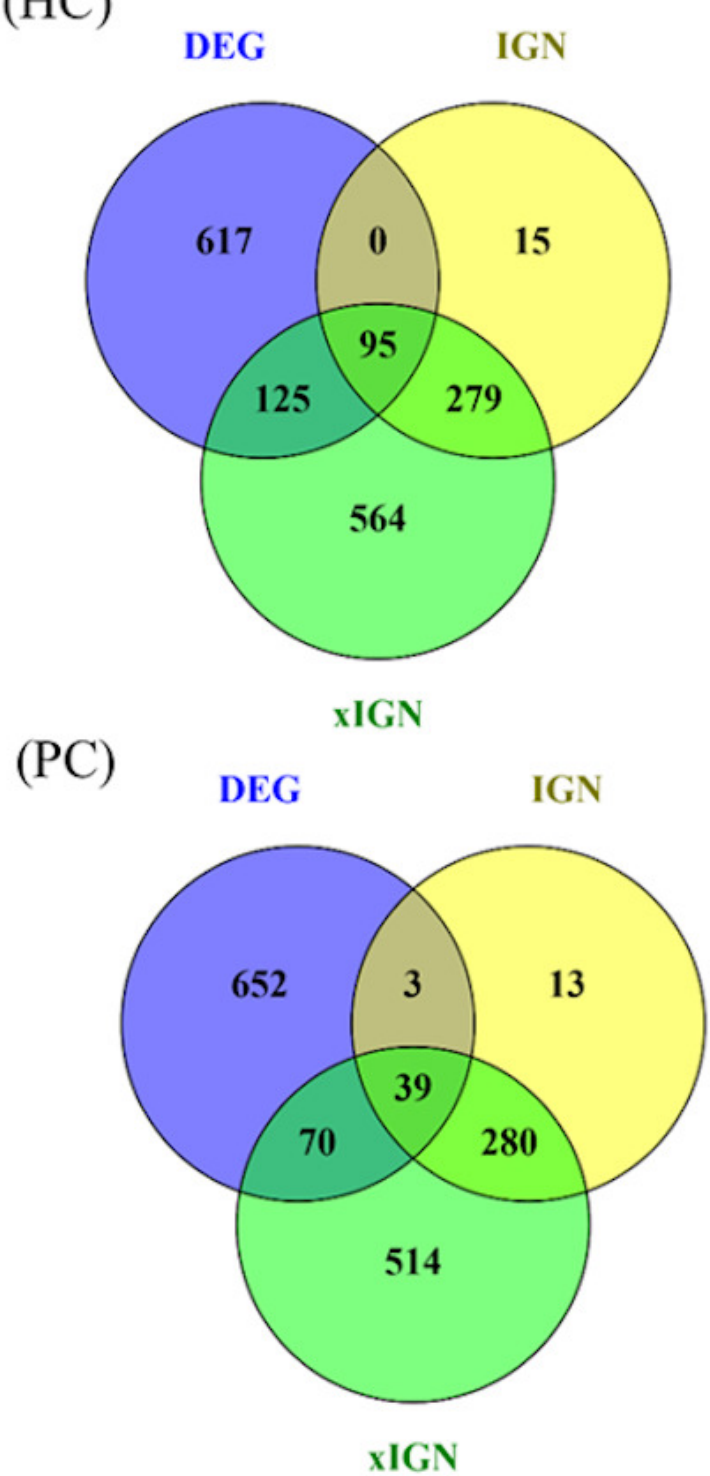

(EC)

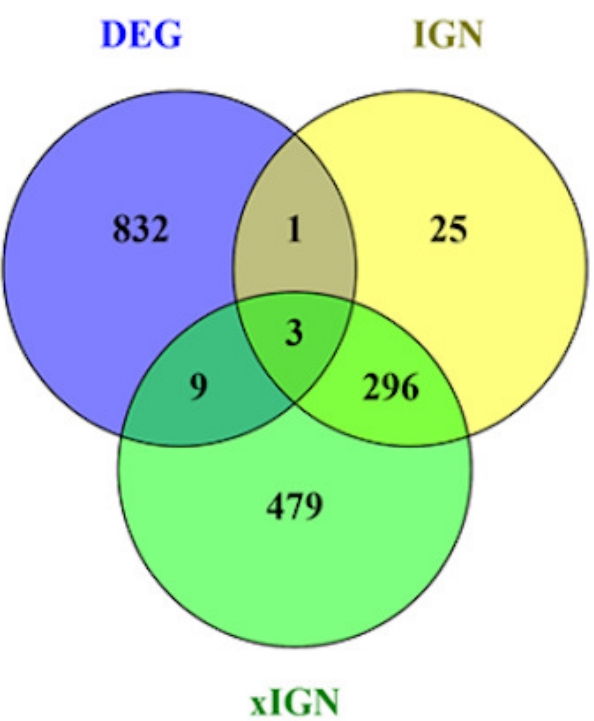

(MTG)

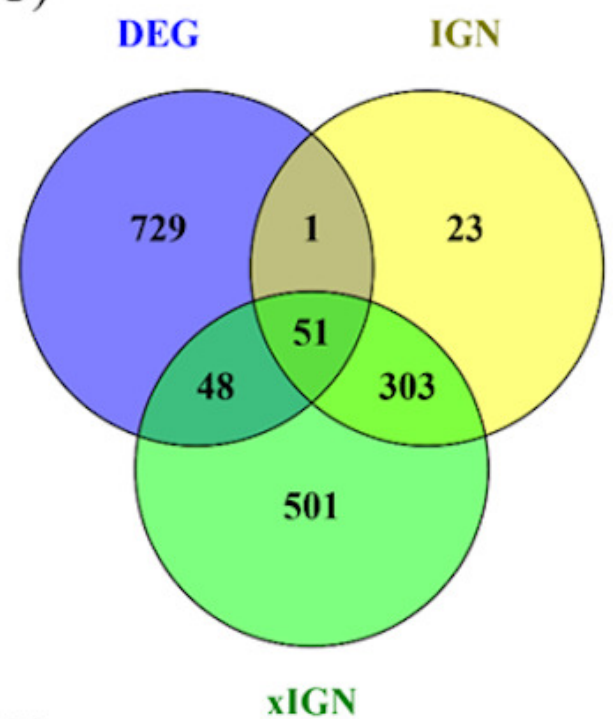

(SFG)

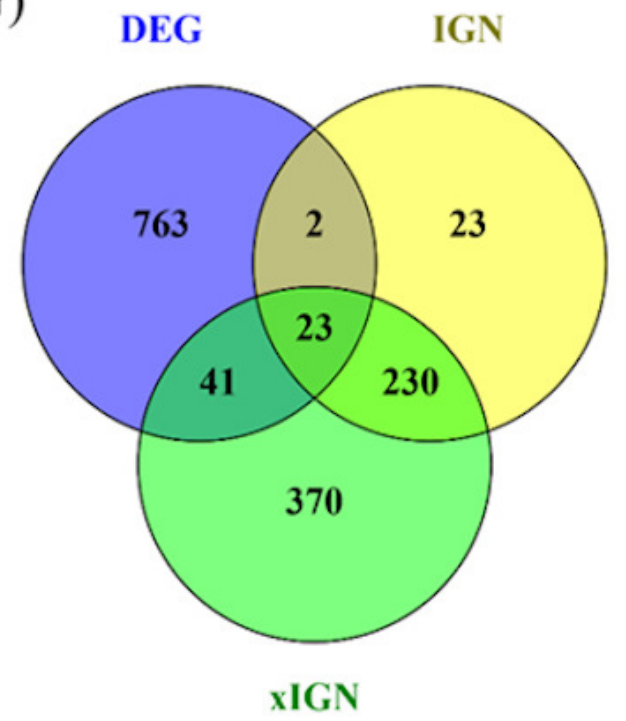




\section{Figure 3}

Enrichment of known AD target genes in curated AG and ALZ gene sets

Top histogram, $-\log _{10}(p)$ (Fisher's exact test $p$-value) of enrichment of top-106 AlzGene genes in curated gene sets. Bottom histogram, enrichment of top-109 AlzBase genes. Abbreviation: AG, normal aging; EC, entorhinal cortex; HC, hippocampus; MTG, medial temporal gyrus; PC, posterior cingulate; SFG, superior frontal gyrus.

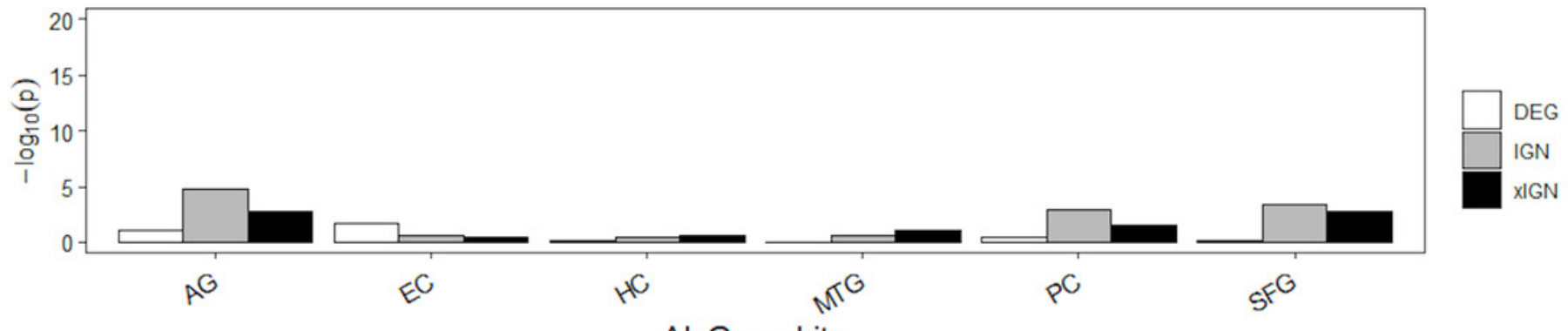

AlzGene hits

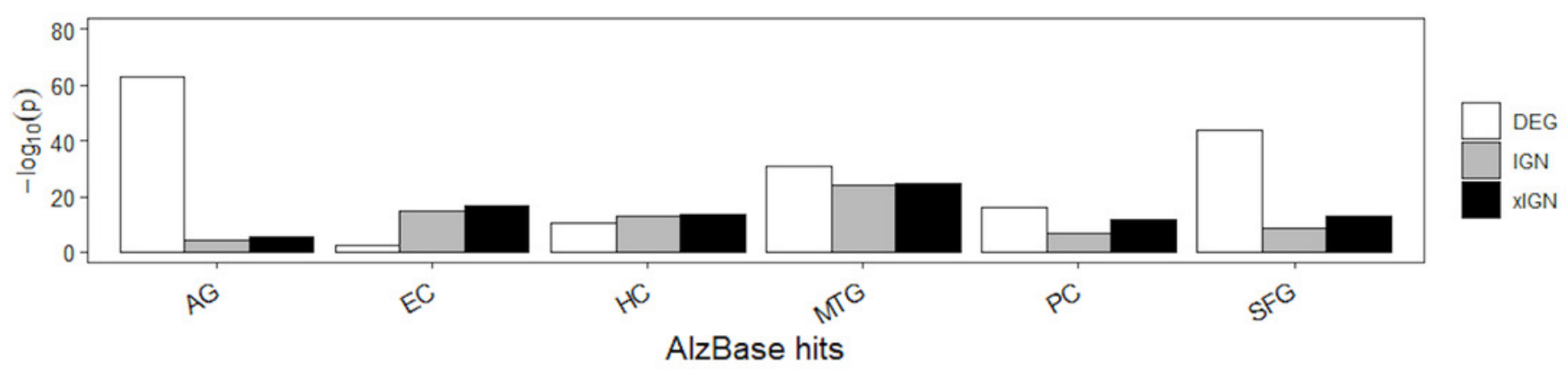




\section{Figure 4}

KEGG pathways most enriched in curated DEG and IGN gene sets

KEGG pathways listed are top-10 by $-\log _{10}(\mathrm{p})$-value. Black (red) towers are for DEG (IGN) enrichment. See Supplementary Tables 12-13 for further details of enriched KEGG pathways in DEG, IGN, and XIGN gene sets. Categories of enriched KEGG pathways are indicated by color bars to the left of the histogram plot: dark gray, cell proliferation; green, neurodegeneration; brown, protein complexes and metabolism; cyan, carcinoma; dark blue, inflammation; orange, metabolic related pathways. Abbreviation: AG, normal aging; EC, entorhinal cortex; HC, hippocampus; MTG, medial temporal gyrus; PC, posterior cingulate; SFG, superior frontal gyrus. A larger list of enriched KEGG pathways are given in Supplementary Tables 12-13. 


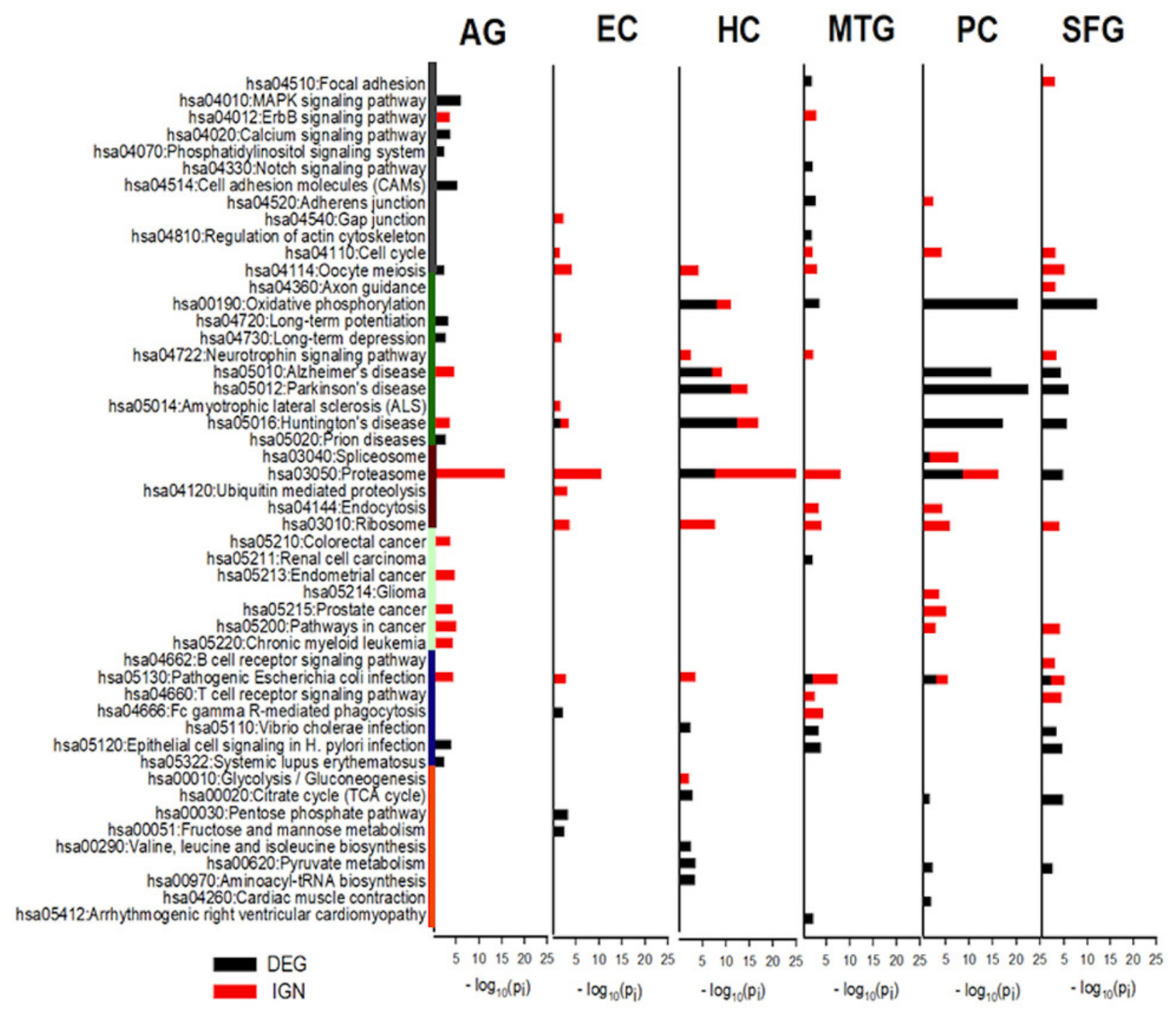




\section{Figure 5}

Volcano plots of ALZ-only hits from six DEG cases

DEGs (cyan, downregulated; light brown, upregulated) were selected using LIMMA with case dependent FDR (for AG, 7.50E-04; EC, 4.0E-06; HC, 1.0e-05; MTG, 5.0e-06; PC, 1.0e-04; SFG, 1.25e-05) and $|\mathrm{FC}(\log 2)|>1$ (red dashline). Dots are "ALZ-only" hits (Table 2 ) in the gene list of leading KEGG pathways enriched in DEGs, including OXPHOS (deep-blue), Proteasome (red), and others (pink). Abbreviation: AG, normal aging; EC, entorhinal cortex; HC, hippocampus; MTG, medial temporal gyrus; PC, posterior cingulate; SFG, superior frontal gyrus; FDR, false discovery rate. 

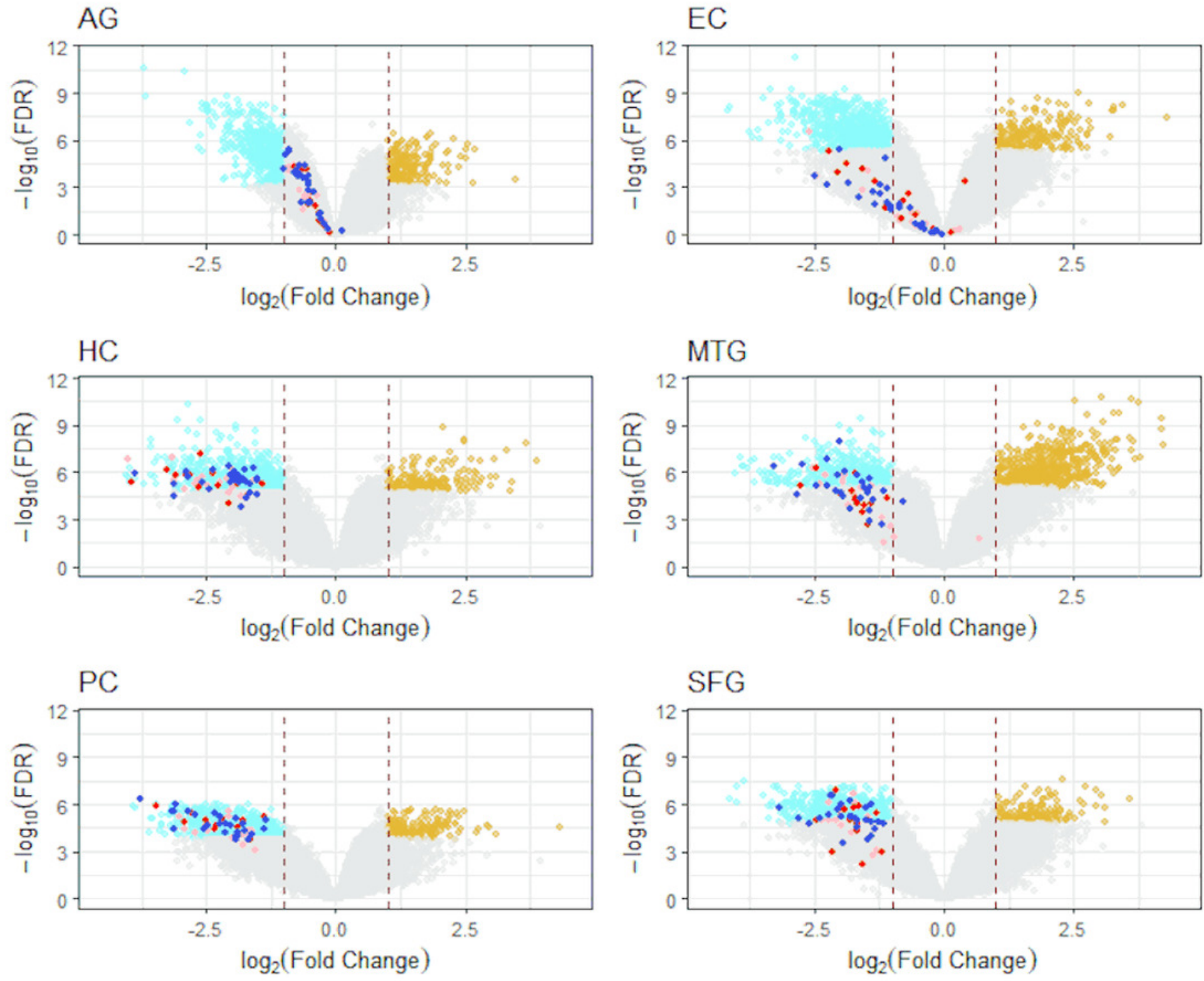


\section{Figure 6}

Enrichment of curated DEG sets in five key KEGG pathways

In the tower labeled "AD+ALZ-only genes", the gene list of the KEGG AD pathway was augmented with 32 of the 54 ALZ-only genes not already in the list, 14 of which are from the pathway Proteasome. Abbreviation: OXPHOS, hsa00190: Oxidative phosphorylation;

Proteasome, hsa03050: Proteasome; PD, hsa05012: Parkinson's disease; HD, hsa05016:

Huntington's disease; AD, hsa05010: Alzheimer's disease; AG, normal aging; EC, entorhinal cortex; HC, hippocampus; MTG, medial temporal gyrus; PC, posterior cingulate; SFG, superior frontal gyrus.

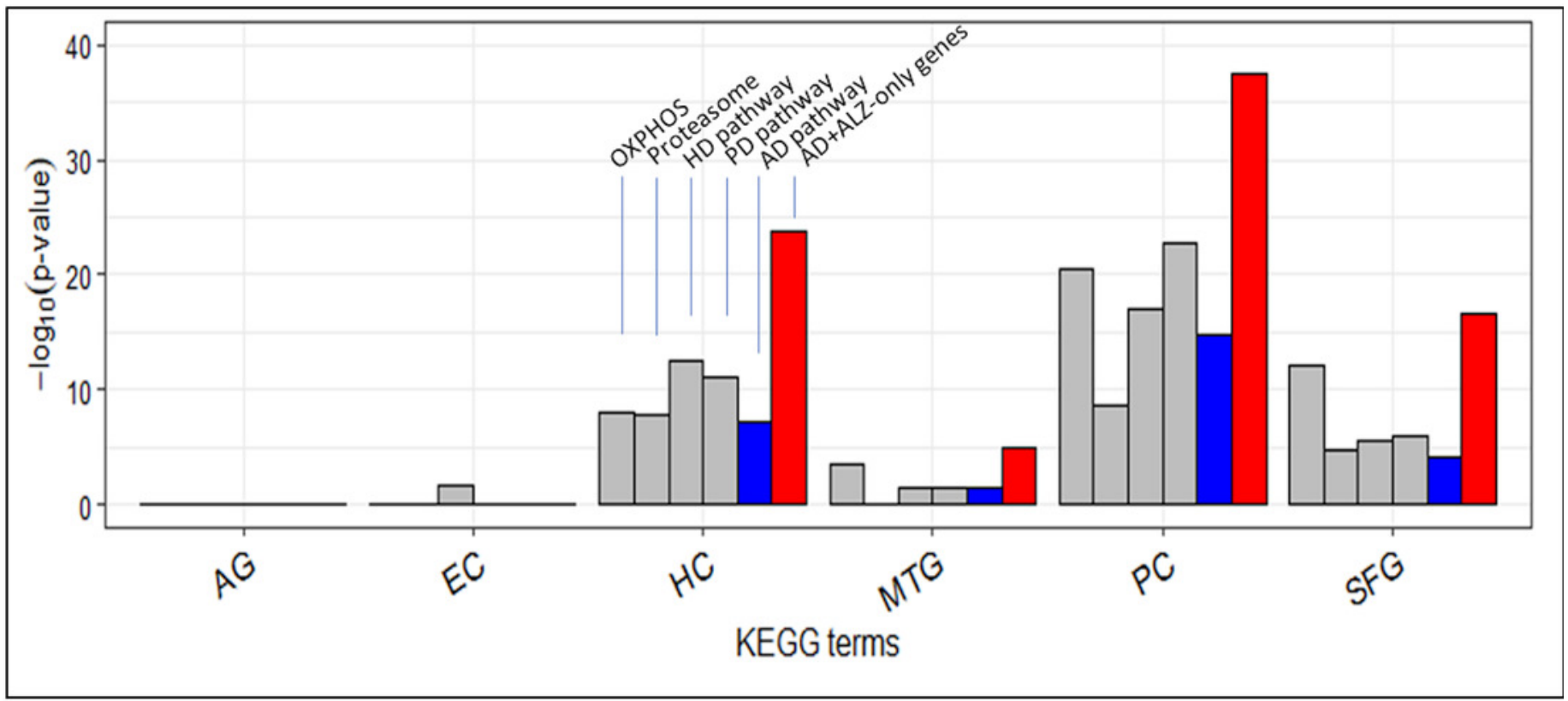




\section{Table $\mathbf{1}$ (on next page)}

Top-5 genes from AlzGene and AlzBase in the curated gene sets

The top-5 genes are the five genes in each of AlzGene and AlzBase having the highest appearance frequencies. In column 2 (DEG) and 3 (IGN), datasets in which the gene occurs (when it does) are given. Number in brackets gives log2 fold change (down-regulated if negative) in the case of DEG and degree (only genes with degree 2 or greater are included). None of the top- 5 genes appear in any of the gene lists of the three KEGG pathways hsa05130: E. coli infection, hsa00190: oxidative phosphorylation (OXPHOS), and hsa03050: Proteasome. Abbreviations for the six cases: EC, entorhinal cortex; HC, hippocampus; MTG, medial temporal gyrus; PC, posterior cingulate; SFG, superior frontal gyrus. Symbols: *, genes were also used as housekeeping genes in AD studies; \#, gene appears in both AlzGene and AlzBase sets. 
1 Table 1.

\begin{tabular}{|c|c|c|c|}
\hline \multicolumn{4}{|c|}{ ALZBASE } \\
\hline \multirow{2}{*}{$\begin{array}{c}\text { GENE } \\
\text { SYMBOL }\end{array}$} & \multicolumn{2}{|c|}{ Curated AG and ALZ gene sets } & \multirow{2}{*}{ Ref } \\
\hline & DEG & IGN & \\
\hline$N S F$ & AG (-1.31), MTG (-3.20), SFG (-2.88) & $\begin{array}{l}\text { EC (4), HC (3), MTG } \\
(10), \text { SFG (2) }\end{array}$ & [73] \\
\hline$N E C A P 1$ & AG (-1.13), HC (-2.36), PC (-2.46), SFG (-2.75) & $\mathrm{AG}(2), \mathrm{EC}(2), \mathrm{HC}(3)$ & {$[74]$} \\
\hline $\mathrm{MDH} 1$ & AG (-1.25), MTG (-3.72), PC (-2.96), SFG (-3.14) & $\mathrm{EC} \mathrm{(3),} \mathrm{HC} \mathrm{(6),} \mathrm{MTG} \mathrm{(4)}$ & {$[75]$} \\
\hline$A M P H$ & AG (-1.31), MTG (-3.43), SFG (-2.65) & AG (3), EC (3), MTG (4) & {$[76]$} \\
\hline$* P G K 1$ & AG (-1.22), HC (-2.71), PC (-2.40) & $\mathrm{EC}(2), \mathrm{HC}(5), \mathrm{MTG}(2)$ & [77] \\
\hline \multicolumn{4}{|c|}{ ALZGENE } \\
\hline${ }^{*} G A P D H$ & $\mathrm{HC}(-3.88), \mathrm{PC}(-3.39)$ & $\mathrm{HC}(5), \mathrm{MTG}(4), \mathrm{PC}(6)$ & {$[78]$} \\
\hline$U B Q L N 1$ & AG $(-1.17)$ & $\mathrm{AG}(3), \mathrm{EC}(5), \mathrm{HC}(4)$ & {$[79]$} \\
\hline$A B C A 1$ & AG (1.32), EC (1.61), MTG (2.21) & - & {$[80]$} \\
\hline${ }^{\#} C D K 5$ & $\mathrm{EC}(-2.20), \quad$ SFG $(-1.80)$ & $\mathrm{PC}(2)$ & {$[81]$} \\
\hline GSK3B & $\mathrm{HC}(-1.75)$ & AG (2), MTG (5) & {$[82]$} \\
\hline
\end{tabular}

2 


\section{Table 2 (on next page)}

Fifty-four AD-only hits and 32 novel $A D$ genes

The 54 AD-only hits were enrichment hits from 6 KEGG pathways - OXPHOS (Oxp), Proteasome (PSM), Pyruvate metabolism (Pym), TCA cycle (TCA), V. cholerae infection (Vch) and Pathogenic E. coli infection (Ecol) - in two of three DEG sets of HC/PC/SFG and not in AG. The 32 genes not in the KEGG AD pathway (AD) are called "novel AD genes". Gene families: acylphosphatase (ACYP), Actin Related Protein 2/3 Complex (ARPC), adenosine triphosphate (ATP), cyclooxygenase (COX), $\alpha$-ketoglutarate dehydrogenase (DLD), fumarate hydratase $(\mathrm{FH})$, glyoxalase (GLO), isocitrate dehydrogenase (IDH), lactate dehydrogenase (LDH), malate dehydrogenase (MDH), NADH:ubiquinone oxidoreductase (NDU), pyruvate dehydrogenase $(\mathrm{PDH})$, proteasome maturation protein (POMP), proteasome subunits (PSM), succinate dehydrogenase (SDH), ubiquinol-cytochrome $c$ reductase (UQCR). Abbreviations for the six cases: EC, entorhinal cortex; HC, hippocampus; MTG, medial temporal gyrus; PC, posterior cingulate; SFG, superior frontal gyrus. 


\section{Table 2.}

2

\begin{tabular}{|c|c|c|c|c|c|c|}
\hline KEGG & $\begin{array}{l}\text { Protein } \\
\text { families }\end{array}$ & Gene & DEG & $\begin{array}{l}\text { Up or } \\
\text { Down }\end{array}$ & IGN & Reference \& Remark \\
\hline \multirow{5}{*}{ Ecol } & ARPC & $A R P C 1 A$ & $\begin{array}{l}\text { HC, PC, } \\
\text { SFG }\end{array}$ & Down & - & $\begin{array}{l}\text { Arp } 2 / 3 \text { complex is a central player in } \\
\text { actin-based motility of pathogens. }\end{array}$ \\
\hline & \multirow{4}{*}{ Tubulin } & $T U B A 1 B$ & $\begin{array}{l}\text { HC, PC, } \\
\text { SFG, MTG }\end{array}$ & Down & $\begin{array}{l}\text { AG, EC, } \\
\text { HC, MTG, } \\
\text { PC, SFG }\end{array}$ & \multirow{4}{*}{$\begin{array}{l}\text { Level of E. coli K99 was reported to } \\
\text { be greater in AD compared to control } \\
\text { brains [66]. Reduced } \alpha \text {-tubulin } \\
\text { expression led to increased human- } \\
\text { tau expression in transgenic worm } \\
\text { [69]. }\end{array}$} \\
\hline & & TUBA1C & $\begin{array}{l}\mathrm{HC}, \mathrm{PC}, \\
\mathrm{MTG}\end{array}$ & Down & $\begin{array}{l}\text { AG, HC, } \\
\text { MTG, PC, } \\
\text { SFG }\end{array}$ & \\
\hline & & $T U B B$ & $\begin{array}{l}\mathrm{HC}, \mathrm{PC}, \\
\mathrm{SFG}\end{array}$ & Down & $\begin{array}{l}\text { AG, EC, } \\
\text { HC, MTG, } \\
\text { PC, SFG }\end{array}$ & \\
\hline & & TUBB3 & $\begin{array}{l}\text { PC, SFG, } \\
\text { MTG }\end{array}$ & Down & $\begin{array}{l}\text { AG, EC, } \\
\text { MTG }\end{array}$ & \\
\hline \multirow{3}{*}{ Pym } & ACYP & $A C Y P 2$ & $\mathrm{HC}, \mathrm{PC}$ & Down & - & \\
\hline & GLO & GLO1 & $\begin{array}{l}\text { HC, PC, } \\
\text { MTG }\end{array}$ & Down & - & \\
\hline & LDH & $L D H B$ & $\mathrm{HC}, \mathrm{PC}$ & Down & - & $\begin{array}{l}\text { Decreased LDHB relative to LDHA } \\
\text { leads to CNS aging in transgenic } \\
\text { mice [63]. }\end{array}$ \\
\hline \multirow{4}{*}{$\begin{array}{l}\text { Pym, } \\
\text { TCA }\end{array}$} & DLD & $D L D$ & $\begin{array}{l}\text { HC, PC, } \\
\text { SFG }\end{array}$ & Down & $\mathrm{HC}, \mathrm{PC}$ & $\begin{array}{l}\text { DLD activity down in AD-human } \\
\text { brain [62]. }\end{array}$ \\
\hline & $\mathrm{MDH}$ & $\mathrm{MDH} 2$ & $\mathrm{HC}, \mathrm{SFG}$ & Down & $\mathrm{EC}$ & $\begin{array}{l}\text { MDH activity up in AD-human brain } \\
\text { [62]. }\end{array}$ \\
\hline & \multirow{2}{*}{ PDH } & PDHAl & $\mathrm{HC}, \mathrm{SFG}$ & Down & - & \multirow{2}{*}{$\begin{array}{l}\text { PDH activity down in AD-human } \\
\text { brain [62] and transgenic mice [61]. }\end{array}$} \\
\hline & & $P D H B$ & $\mathrm{PC}, \mathrm{SFG}$ & Down & $\mathrm{HC}, \mathrm{SFG}$ & \\
\hline \multirow{2}{*}{ TCA } & $\mathrm{FH}$ & $F H$ & $\begin{array}{l}\text { PC, SFG, } \\
\text { MTG }\end{array}$ & Down & - & $\begin{array}{l}\text { Activity changed in AD-human brain } \\
{[62] \text {. }}\end{array}$ \\
\hline & IDH & $I D H 3 G$ & $\begin{array}{l}\text { EC, HC, } \\
\text { SFG }\end{array}$ & Down & - & $\begin{array}{l}\text { IDH activity down in AD-human } \\
\text { brain [62]. }\end{array}$ \\
\hline $\begin{array}{l}\text { TCA, } \\
\text { Oxp, } \\
\text { AD }\end{array}$ & SDH & $S D H A$ & $\mathrm{HC}, \mathrm{PC}$ & Down & $\mathrm{AG}$ & $\begin{array}{l}\text { SDH activity down in transgenic } \\
\text { mice [61]. }\end{array}$ \\
\hline \multirow{2}{*}{$\begin{array}{l}\text { Vch, } \\
\text { Oxp }\end{array}$} & \multirow{2}{*}{ ATP } & ATP6V1E1 & $\begin{array}{l}\text { HC, PC, } \\
\text { SFG, MTG }\end{array}$ & Down & $\begin{array}{l}\text { EC, HC, } \\
\text { MTG }\end{array}$ & \\
\hline & & ATP6V1H & $\mathrm{HC}, \mathrm{PC}$ & Down & $\begin{array}{l}\text { EC, HC, } \\
\text { MTG }\end{array}$ & \\
\hline \multirow{2}{*}{ Oxp } & \multirow{2}{*}{ ATP } & ATP5J2 & $\begin{array}{l}\text { PC, SFG, } \\
\text { EC, MTG }\end{array}$ & Down & - & \multirow{6}{*}{$\begin{array}{l}\text { ATP activity down in transgenic } \\
\text { mice [45]. }\end{array}$} \\
\hline & & $A T P 5 L$ & $\begin{array}{l}\text { PC, SFG, } \\
\text { MTG }\end{array}$ & Down & & \\
\hline \multirow{4}{*}{$\begin{array}{l}\text { Oxp } \\
\text { AD }\end{array}$} & \multirow{4}{*}{ ATP } & ATP 5A1 & $\mathrm{HC}, \mathrm{PC}$ & Down & $\begin{array}{l}\text { EC, HC, } \\
\text { SFG }\end{array}$ & \\
\hline & & $A T P 5 B$ & $\begin{array}{l}\mathrm{HC}, \mathrm{PC}, \\
\text { SFG }\end{array}$ & Down & $\begin{array}{l}\text { EC, HC, } \\
\text { MTG, SFG }\end{array}$ & \\
\hline & & $A T P 5 C 1$ & $\begin{array}{l}\text { PC, SFG, } \\
\text { MTG }\end{array}$ & Down & $\begin{array}{l}\text { EC, HC, } \\
\text { MTG, SFG }\end{array}$ & \\
\hline & & ATP5G3 & $\mathrm{HC}, \mathrm{PC}$, & Down & - & \\
\hline
\end{tabular}




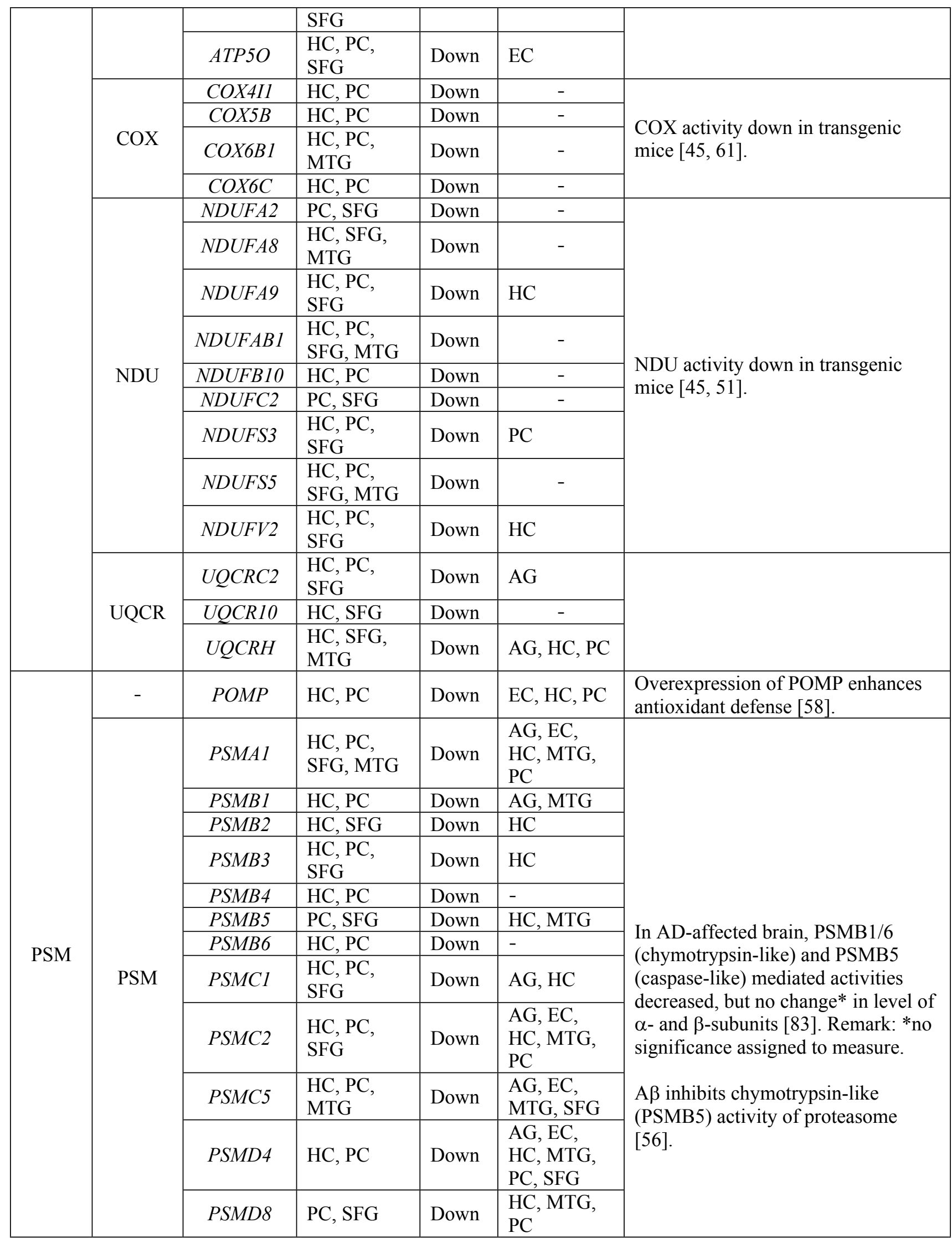


3

4

Peer) reviewing PDF | (2019:08:39955:1:0:NEW 13 Jan 2020) 